\title{
A metallicity study of 1987A-like supernova host galaxies ${ }^{\star \star \star}$
}

\author{
F. Taddia ${ }^{1}$, J. Sollerman ${ }^{1}$, A. Razza ${ }^{1}$, E. Gafton ${ }^{1}$, A. Pastorello ${ }^{2}$, C. Fransson ${ }^{1}$, M. D. Stritzinger ${ }^{3}$, \\ G. Leloudas ${ }^{4,5}$, and M. Ergon ${ }^{1}$
}

\author{
1 The Oskar Klein Centre, Department of Astronomy, Stockholm University, AlbaNova, 10691 Stockholm, Sweden \\ e-mail: francesco.taddia@astro.su.se \\ 2 INAF - Osservatorio Astronomico di Padova, Vicolo dellOsservatorio 5, 35122 Padova, Italy \\ 3 Department of Physics and Astronomy, Aarhus University, Ny Munkegade 120, 8000 Aarhus C, Denmark \\ 4 The Oskar Klein Centre, Department of Physics, Stockholm University, AlbaNova, 10691 Stockholm, Sweden \\ 5 Dark Cosmology Centre, Niels Bohr Institute, University of Copenhagen, Juliane Maries Vej 30, 2100 Copenhagen, Denmark
}

Received 14 July 2013 / Accepted 26 August 2013

\section{ABSTRACT}

Context. The origin of the blue supergiant (BSG) progenitor of Supernova (SN) 1987A has long been debated, along with the role that its sub-solar metallicity played. We now have a sample of SN 1987A-like events that arise from the rare core collapse (CC) of massive $\left(\sim 20 M_{\odot}\right)$ and compact $\left(\lesssim 100 R_{\odot}\right)$ BSGs.

Aims. The metallicity of the explosion sites of the known BSG SNe is investigated, as well as the association of BSG SNe to starforming regions.

Methods. Both indirect and direct metallicity measurements of 13 BSG SN host galaxies are presented, and compared to those of other CC SN types. Indirect measurements are based on the known luminosity-metallicity relation and on published metallicity gradients of spiral galaxies. In order to provide direct metallicity measurements based on strong line diagnostics, we obtained spectra of each BSG SN host galaxy both at the exact SN explosion sites and at the positions of other H II regions. We also observed these hosts with narrow $\mathrm{H} \alpha$ and broad $R$-band filters in order to produce continuum-subtracted $\mathrm{H} \alpha$ images. This allows us to measure the degree of association between BSG SNe and star-forming regions, and to compare it to that of other SN types.

Results. BSG SNe are found to explode either in low-luminosity galaxies or at large distances from the nuclei of luminous hosts. Therefore, their indirectly measured metallicities are typically lower than those of SNe IIP and Ibc. This result is confirmed by the direct metallicity estimates, which show slightly sub-solar oxygen abundances $(12+\log (\mathrm{O} / \mathrm{H}) \sim 8.3-8.4$ dex $)$ for the local environments of BSG SNe, similar to that of the Large Magellanic Cloud (LMC), where SN 1987A exploded. However, we also note that two objects of our sample (SNe 1998A and 2004em) were found at near solar metallicity. SNe IIb have a metallicity distribution similar to that of our BSG SNe. Finally, we find that the degree of association to star-forming regions is similar among BSG SNe, SNe IIP and IIn.

Conclusions. Our results suggest that LMC metal abundances play a role in the formation of some 1987A-like SNe. This would naturally fit in a single star scenario for the progenitors. However, the existence of two events at nearly solar metallicity suggests that also other channels, e.g. binarity, contribute to produce BSG SNe.

Key words. supernovae: general - stars: evolution - galaxies: abundances

\section{Introduction}

Supernova (SN) 1987A, the most studied SN ever, was also the first object whose progenitor (Sanduleak $-69^{\circ} 202$ ) could be directly identified (Gilmozzi et al. 1987). Surprisingly, the massive $\left(M_{\text {ZAMS }} \sim 16-22 M_{\odot}\right.$, Arnett et al. 1989) progenitor star was not a red supergiant (RSG) with a radius of a few hundred $R_{\odot}$ as envisioned by contemporary theory, but rather a compact $\left(R \lesssim 50 R_{\odot}\right)$ blue supergiant (BSG). The compactness of its progenitor explained both its slowly rising light curve $(\approx 84$ days $)$ and its low luminosity in the first months (e.g. Arnett et al. 1989). Most of the kinetic energy was spent to adiabatically expand the star while the light curve of SN 1987A was mainly powered by the radioactive decay of ${ }^{56} \mathrm{Ni}$ and ${ }^{56} \mathrm{Co}$.

^ Based on observations performed at the Nordic Optical Telescope (Proposal number 44-011 and 45-004, PI: F. Taddia; and 47-701), La Palma, Spain; the Very Large Telescope (Program 090.D-0092, PI: F. Taddia), Paranal, Chile. Some data were also obtained from the ESO Science Archive Facility and from the Isaac Newton Telescope archive. $\star \star$ Figures 4-14 are available in electronic form at http://www . aanda.org
Many attempts have been made to explain the nature of the BSG progenitor of SN 1987A (see Podsiadlowski 1992 for a review), since standard stellar models did not predict the explosion of massive stars in the BSG phase (e.g. Woosley et al. 2002). As a matter of fact, a single non-rotating $\sim 20 M_{\odot}$ star born in a solar-metallicity environment is expected to be a RSG at the time of collapse (Eldridge 2005). Therefore, special conditions are required for a massive star to be a BSG at the endpoint of its life. The possible scenarios include those that involve a single progenitor star and those that consider Sanduleak $-69^{\circ} 202$ as part of a binary system. We note that for the specific case of the progenitor of SN 1987A, any proposed scenario not only has to explain why it was a BSG, but must also fit other constraints such as the chemical anomalies (Saio et al. 1988; Fransson et al. 1989) and the peculiar CSM of SN 1987A (Morris \& Podsiadlowski 2009). We now briefly review the possible mechanisms that lead to the explosion of a BSG according to theoretical models.

In the single-star framework, a first natural explanation for the BSG nature of SN 1987A's progenitor was found in the subsolar metallicity $(12+\log (\mathrm{O} / \mathrm{H})=8.37 \mathrm{dex}$, Russell \& Dopita 1990) measured in the Large Magellanic Cloud (LMC), which 
hosted SN 1987A (Langer 1991). A low metallicity can allow a blue solution in the HR diagram for the endpoint of a massive star (see e.g., Brunish \& Truran 1982; Arnett 1987; Hillebrandt et al. 1987; Woosley 1988). Another single star scenario that can lead to the explosion of a BSG is that invoking a rotating star, where rotational mixing plays an important role in pushing the stellar evolution towards the blue part of the HR diagram (see e.g. Weiss et al. 1988; Hirschi et al. 2004; Ekström et al. 2012).

In the binary scenario, both accretion and merger models may lead to the explosion of BSGs (see Woosley et al. 2002, for references). We note that the binary solution was considered for the specific case of SN 1987A because it would more easily explain the "hour-glass" shape of the CSM observed in its remnant (Morris \& Podsiadlowski 2009). As noted by Woosley et al. (2002), if massive stars end their lives as BSGs because they merge with a companion, we would expect to find BSG SNe also at solar metallicity.

From the observational point of view, testing the role of metallicity in the production of exploding BSGs has not been possible until recently, because SN 1987A belonged to a class of its own for many years, although a handful of poorly observed $\mathrm{SNe}$ were suggested to be similar to it already in the months following the explosion of SN 1987A (see e.g. van den Bergh 1988). Among these SNe, following Pastorello et al. (2012), we consider only SNe 1909A and 1982F as reliable 1987A-like transients. Therefore, it was impossible to say whether the explosion of compact BSGs usually occurs at low metallicity or not.

Recently, however, two new SNe with BSG progenitors were presented by Kleiser et al. (2011). These objects, SNe 2000cb and 2005ci, display a long rise time and low luminosity at early epochs, closely resembling the light curve of SN 1987A. The BSG origin of SN 2000cb was later corroborated by detailed modeling (Utrobin \& Chugai 2011). Before these events, SN 1998A (Pastorello et al. 2005) had been suggested to result from the explosion of a BSG star. Recently we published an analysis of two additional SN 1987A-like transients (SNe 2006V and 2006au; Taddia et al. 2012). Through the modeling of the bolometric light curves, we determine progenitor radii to less than $100 R_{\odot}$. This clearly argues for compact BSG progenitors. Furthermore, in Pastorello et al. (2012), we presented another object, SN 2009E, which also has a BSG origin. Pastorello et al. (2012) discussed the sample of known SN 1987A-like objects, listing a total of 11 transients including the aforementioned SNe. Arcavi et al. (2012) added a few new examples to the family of known SN 1987A-like events. The complete list of BSG SNe is given in Table 1, where we also report basic information concerning their hosts, i.e. the coordinates, morphological type, axis dimensions, position angle, absolute magnitude, Milky Way color excess, redshift and distance modulus.

This new sample of BSG SNe allows us to investigate the role played by the metallicity in the origin of BSG explosions. Studying the metallicity of SN host galaxies has become a popular line of investigation. Indirect estimates based on galaxy luminosity (Tremonti et al. 2004) and known metallicity gradients (e.g. Pilyugin et al. 2004) are useful indicators, but this sort of analysis can be substantially improved by determining the metallicities at the exact sites of the explosion long after the $\mathrm{SN}$ faded away (e.g. Leloudas et al. 2011; Modjaz et al. 2011) and the actual metallicity gradient through emission line diagnostics (e.g. Thöne et al. 2009).

The aim of this paper is to provide an observational test of the role of metallicity for BSG SNe, and we therefore perform a detailed metallicity analysis of the galaxies that hosted a BSG SN.
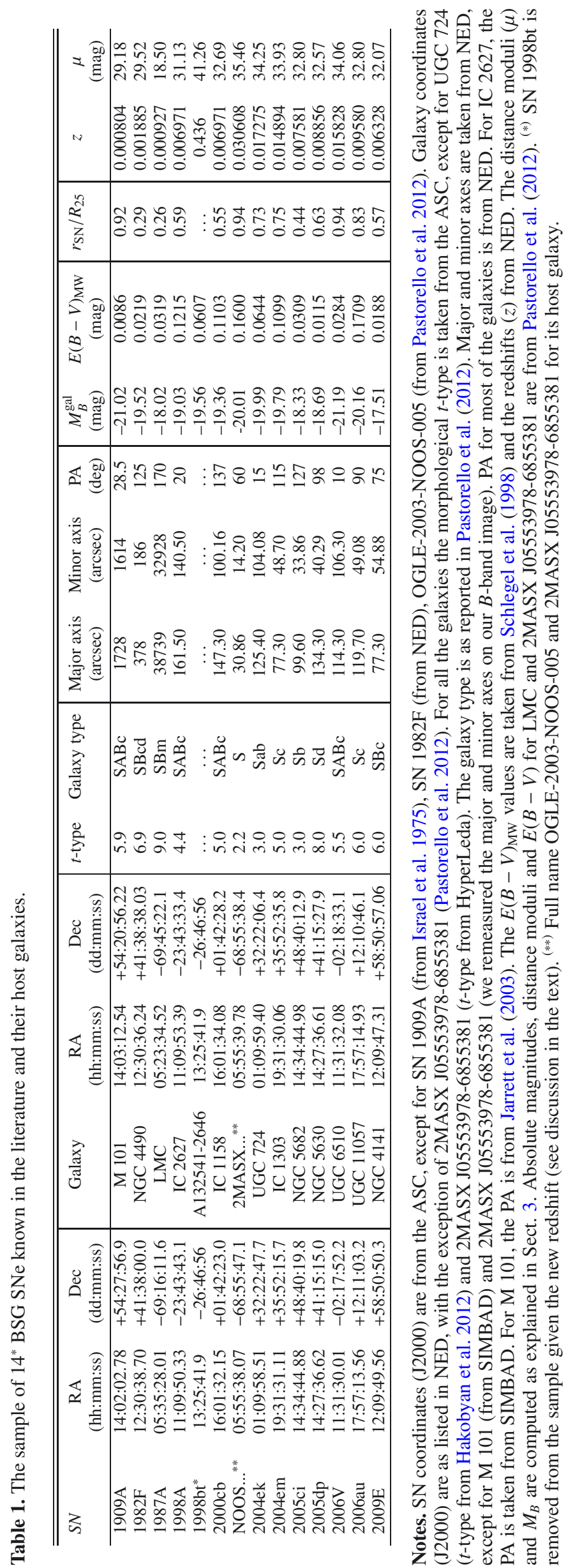
Table 2. Log of the photometric and spectroscopic observations.

\begin{tabular}{|c|c|c|c|c|c|c|c|}
\hline \multirow[t]{2}{*}{ SN } & \multirow[t]{2}{*}{ Galaxy } & \multicolumn{3}{|c|}{ Photometry } & \multicolumn{3}{|c|}{ Spectroscopy } \\
\hline & & Telescope & Instrument+Filter & Integration (s) & Telescope & Instrument+Grism+Slit & Integration (s) \\
\hline \multirow[t]{2}{*}{$1909 \mathrm{~A}$} & M 101 & NOT & ALFOSC+R* & $3 \times 200$ & NOT & ALFOSC+\#4+1.0" & $1 \times 1800$ \\
\hline & & NOT & $\mathrm{ALFOSC}+\mathrm{H} \alpha(\# 21)^{*}$ & $3 \times 40$ & & & \\
\hline \multirow[t]{2}{*}{$1982 \mathrm{~F}$} & NGC 4490 & INT & $\mathrm{WFC}+\mathrm{R}$ & $1 \times 200$ & NOT & ALFOSC+\#4+1.0" & $1 \times 1800$ \\
\hline & & INT & $\mathrm{WFC}+\mathrm{H} \alpha(\# 197)$ & $1 \times 750$ & & & \\
\hline 1998A & IC 2627 & VLT & $\mathrm{FORS} 2+\mathrm{R}$ & $5 \times 10$ & VLT & FORS $2+300 \mathrm{~V}+1.0^{\prime \prime}$ & $3 \times 600$ \\
\hline \multirow[t]{3}{*}{ (1994R) } & & VLT & FORS $2+\mathrm{H} \alpha / 2500+60$ & $5 \times 80$ & VLT & FORS $2+300 \mathrm{~V}+1.0^{\prime \prime}$ & $3 \times 600$ \\
\hline & & & & & NTT & EMMI +\#3+1.5" & $2 \times 300$ \\
\hline & & & & & NTT & EMMI+\#3+1.5" & $2 \times 300$ \\
\hline \multirow[t]{2}{*}{ 1998bt } & A132541-2646 & VLT & $\mathrm{FORS} 2+\mathrm{R}$ & $3 \times 150$ & VLT & FORS2+300V+1.0" & $3 \times 1800$ \\
\hline & & VLT & $\mathrm{FORS} 2+\mathrm{B}$ & $3 \times 300$ & & & \\
\hline \multirow[t]{2}{*}{$2000 \mathrm{cb}$} & IC 1158 & VLT & FORS2+R & $10 \times 10$ & VLT & FORS2+300V+1.0" & $3 \times 1200$ \\
\hline & & VLT & FORS $2+\mathrm{H} \alpha / 2500+60$ & $10 \times 80$ & & & \\
\hline \multirow[t]{3}{*}{ NOOS...* } & 2MASX...* & VLT & FORS $2+\mathrm{R}$ & $2 \times 10+3 \times 20$ & VLT & FORS $2+300 \mathrm{~V}+1.0^{\prime \prime}$ & $4 \times 900$ \\
\hline & & VLT & FORS2+SII 2000(+63) & $5 \times 80$ & & & \\
\hline & & VLT & FORS2+B & $2 \times 20+3 \times 10$ & & & \\
\hline \multirow[t]{2}{*}{ 2004em } & IC 1303 & NOT & $\mathrm{ALFOSC}+\mathrm{R}$ & $3 \times 40$ & NOT & ALFOSC+\#4+1.0" & $2 \times 1800$ \\
\hline & & NOT & ALFOSC $+\mathrm{H} \alpha(\# 50)$ & $3 \times 200$ & & & \\
\hline \multirow[t]{2}{*}{$2005 \mathrm{ci}$} & NGC 5682 & NOT & $\mathrm{ALFOSC}+\mathrm{R}$ & $3 \times 40$ & NOT & ALFOSC+\#4+1.0" & $3 \times 1800$ \\
\hline & & NOT & ALFOSC $+\mathrm{H} \alpha(\# 77)$ & $3 \times 200$ & NOT & ALFOSC+\#4+1.0" & $3 \times 1800$ \\
\hline \multirow[t]{3}{*}{$2005 \mathrm{dp}$} & NGC 5630 & NOT & $\mathrm{ALFOSC}+\mathrm{R}$ & $3 \times 300$ & NOT & ALFOSC+\#4+1.0" & $3 \times 1800$ \\
\hline & & NOT & ALFOSC $+\mathrm{H} \alpha(\# 49)$ & $2 \times 600$ & NOT & ALFOSC+\#4+1.0" & $3 \times 1800$ \\
\hline & & & & & NOT & ALFOSC+\#8+1.0" & $1 \times 1800$ \\
\hline \multirow[t]{2}{*}{$2006 \mathrm{~V}$} & UGC 6510 & VLT & $\mathrm{FORS} 2+\mathrm{R}$ & $5 \times 10$ & VLT & FORS $2+300 \mathrm{~V}+1.0^{\prime \prime}$ & $3 \times 1200$ \\
\hline & & VLT & FORS $2+\mathrm{H} \alpha / 4500+61$ & $5 \times 80$ & NOT & ALFOSC+\#4+1.0" & $3 \times 1800$ \\
\hline \multirow[t]{2}{*}{ 2006au } & UGC 11057 & VLT & $\mathrm{FORS} 2+\mathrm{R}$ & $10 \times 5$ & VLT & FORS $2+300 \mathrm{~V}+1.0^{\prime \prime}$ & $3 \times 1200$ \\
\hline & & VLT & FORS $2+\mathrm{H} \alpha / 2500+60$ & $10 \times 40$ & & & \\
\hline \multirow[t]{2}{*}{$2009 \mathrm{E}$} & NGC 4141 & NOT & ALFOSC $+\mathrm{R}$ & $3 \times 40$ & NOT & ALFOSC+\#4+1.0" & $3 \times 1800$ \\
\hline & & NOT & ALFOSC $+\mathrm{H} \alpha(\# 77)$ & $3 \times 200$ & & & \\
\hline
\end{tabular}

Notes. ${ }^{(*)}$ With the NOT we imaged M 101 only within the FOV including the positions of SN 1909A and of the H II region that we spectroscopically observed (see the top panel of Fig. 4). The continuum-subtraced $\mathrm{H} \alpha$ image of the entire galaxy was obtained from http://www. aoc.nrao. edu. ${ }^{(* *)}$ Full name OGLE-2003-NOOS-005 and 2MASX J05553978-6855381 for its host galaxy.

In Sect. 2 details are given on our photometric and spectroscopic observations; Sect. 3 includes the results on the metallicity of the BSG SN explosion sites that were obtained indirectly from the galaxy luminosity and the SN distance from its host center; in Sect. 4 we present a metallicity study of the observed BSG hosts using long slit spectroscopy. This is based on strong line diagnostics, including metallicity estimates at the exact explosion sites and a metallicity gradient for each host that was observed. Section 5 describes the measurements and the results concerning the association of BSG $\mathrm{SNe}$ to star-forming regions in their hosts. A discussion based on these results is given in Sect. 6 and our conclusions follow in Sect. 7.

\section{Observations and data reduction}

A log of all the observations that were performed and collected for this study is presented in Table 2. A total of 14 BSG SNe are known in the literature, including SN 1987A. We gathered photometric and spectroscopic data for 12 of them (the metallicity of SN 1987A is known and we did not observe the host of SN 2004ek, which was very recently identified as a BSG SN by Arcavi et al. 2012). We rejected SN 1998bt from the sample after measuring its redshift, which made this $\mathrm{SN}$ too bright to be a SN 1987A-like event (see Sect. 2.2). Therefore, a total of 11 BSG SN host galaxies were directly investigated to obtain their metallicities.

\subsection{Photometry}

Photometric observations in broad $R$ (Bessel) and narrow $\mathrm{H} \alpha$ passbands were carried out at the Nordic Optical Telescope (NOT) using ALFOSC, during the nights starting on March 29
2012, April 12 and 13, 2012 and May 20 and 22, 2013 for the host galaxies of SNe 2004em, 2005ci, 2005dp and 2009E.

The host galaxies of SNe 1998bt and OGLE-2003-NOOS005 were observed on March 172013 at the UT1 of the Very Large Telescope (VLT), which also provided deep images for the hosts of SNe 1998A, 2000cb, 2006V and 2006au. These images were obtained with FORS2 in broad $R$ and narrow $\mathrm{H} \alpha$ filters. For each galaxy we chose the narrow filter with the effective wavelength matching the redshifted wavelength of $\mathrm{H} \alpha$ (filter names are given in Table 2). For instance, the high-redshift host of OGLE-2003-NOOS-005 was observed with the narrow S II filter to match the observed $\mathrm{H} \alpha$ wavelength. This galaxy was also observed in the $B$ band, as was the host of SN 1998bt; the latter was not observed in $\mathrm{H} \alpha$ given its low luminosity. For the host of SN $1982 \mathrm{~F}$ we used broad $R$ and narrow $\mathrm{H} \alpha$ archive images that were taken with the Isaac Newton Telescope. The continuumsubtracted $\mathrm{H} \alpha$ image of M 101 (host of SN 1909A) was obtained from http: //www. aoc.nrao.edu.

Images obtained with ALFOSC and FORS2 are characterized by a pixel scale of $0.19^{\prime \prime} /$ pix and $0.25^{\prime \prime} /$ pix, respectively. This corresponds to $\approx 0.1 \mathrm{kpc} /$ pix at the distance of $100 \mathrm{Mpc}$.

The total integration times, which are on the order of a few hundred seconds for each galaxy, were subdivided into multiple exposures for each filter. In this way, after bias-subtraction and flat field correction, the combination of multiple frames allowed for the removal of cosmic rays. The WCS coordinates of each final $R$-band image were re-calibrated by matching the point sources in the frame with those in the USNO-B catalogue (Monet et al. 2003). This was done using the software available at http: //astrometry. net and presented by Lang et al. (2010). This step was necessary to identify the pixel corresponding to the $\mathrm{SN}$ position in the galaxy with high precision. 
Armed with the scientific frames in narrow $\mathrm{H} \alpha$ and broad $R$ band, we proceeded to build the continuum-subtracted $\mathrm{H} \alpha$ image for each galaxy. We aligned the images in the two filters (using the task "imalign" in IRAF), then we scaled the fluxes in order to match the counts of the stars in the two frames. Successively, the $R$-band image was subtracted from that obtained with the narrow filter, to remove the continuum and to leave only the $\mathrm{H} \alpha(+[\mathrm{NII}])$ emission component, characteristic of $\mathrm{H}$ II regions. Bright foreground stars sometimes leave residuals after the subtraction, and we patched these imperfections by setting the values of these pixels equal to those of the closest portions of the frame. The resulting $\mathrm{H} \alpha$ maps were useful both to measure the association of BSG SNe to star-forming regions and to show the exact locations of the H II regions that were spectroscopically observed.

We also measured the apparent magnitudes of the host of SN 1998 bt from the $B$ - and $R$-band images, by using stars and galaxies of known magnitudes (from SIMBAD) in the local field. We confirmed $m_{R}=23.30 \pm 0.10 \mathrm{mag}$ (Germany et al. 2004) and found $m_{B}=21.95 \pm 0.10 \mathrm{mag}$.

\subsection{Spectroscopy}

Aiming to directly measure the metallicity for the environment of each BSG SN through strong line diagnostics, we obtained long exposures $(>1800 \mathrm{~s})$ with spectroscopy at their location and simultaneously at the center of their host galaxies. The telescope field was rotated to the angle determined by the host center and the SN position, the slit was aligned on a nearby star in the host galaxy field and then the proper offset was given to intercept both the host nucleus and the explosion site. This procedure minimizes the error on the pointing, and was checked with through-slit images. For SN 1909A, whose position was known to be far from bright $\mathrm{H}$ II regions, we placed the slit on a nearby $\mathrm{H} \alpha$ emitting region, also to check the consistency of our measurements with those already published. We complemented the data in the literature also for SN 1982F, by placing the slit close to the $\mathrm{SN}$ position and through bright $\mathrm{H}$ II regions that were not at the center of the host. For the hosts of SNe 2005ci, 2005dp and 2009E we also considered the spectra included in the Sloan Digital Sky Survey (SDSS) archive ${ }^{1}$ that were taken at the center of each host.

Spectra of the hosts of SNe 1909A, 1982F, 2004em, 2005ci, 2005db, 2006V and 2009E were taken at the NOT (+ ALFOSC with grism \#4), as reported in Table 2 . This particular grism covers the $\sim 3500-9000 \AA$ wavelength range, enabling the detection of the most important H II region lines (see Sect. 4). A slit of 1" width was chosen to match the typical seeing at the NOT and to reach a good compromise between spatial resolution and exposure times. VLT with FORS2, grism $300 \mathrm{~V}$ and a $1^{\prime \prime}$ wide slit was used to get spectra of the SN 1987A-like hosts that were observable from the Southern Hemisphere (SNe 1998A, 1998bt, 2000cb, OGLE-2003-NOOS-005, 2006V, and $2006 \mathrm{au).} \mathrm{The}$ wavelength range covered with this setup is 3300-8650 $\AA$. The full-width-at-half-maximum (FWHM) spectral resolution obtained at the NOT and at the VLT is about 16-17 $\AA$ and $11-14 \AA$, respectively. Archival spectra complemented the dataset for SN 1998A. In this case, we reconstructed the slit position, obtaining a perfect match between the position of bright $\mathrm{H} \alpha$ emitting regions in the 2D-spectra and those observed in the $\mathrm{H} \alpha$ images. Since these archival spectra included the SN signal, the pointing was trivial to establish.

\footnotetext{
Through http://skyserver.sdss3.org
}

The spectral reduction was carried out through the following steps: each 2D-spectrum was a) biased subtracted and b) flat-field corrected to remove CCD inhomogeneities and minimize the fringing in the red part of the spectrum. c) If multiple 2D-spectra were obtained, each 2D-frame was combined to remove cosmic rays. Then d) the trace of the galaxy nucleus or that of a bright star or of a $\mathrm{H}$ II region with a bright continuum was extracted and fit by a low-order polynomial. This trace was plotted over the 2D-spectrum to visually check its precision. e) All the spectra of $\mathrm{H}$ II regions visible in the 2D-spectrum were extracted using the same trace, but shifted in the spatial direction to match their positions. The extraction region was determined by looking at the $\mathrm{H} \alpha$ flux profile and then plotting this region over the 2D-spectrum to ensure the tracing and to include all the emission lines. f) Each spectrum was wavelength calibrated by comparison with a spectrum of an arc-lamp. g) The wavelengthcalibrated spectrum was flux calibrated with the sensitivity function obtained from the spectrum of a standard star observed in the same night. Both the H II region spectra and the standard spectrum were corrected for the airmass and for the second order contamination following the prescriptions of Stanishev (2007).

The spectra that show at least $\mathrm{H} \alpha$ and [N II] $\lambda 6584$ emission were included in this study. The spatial position of the corresponding $\mathrm{H}$ II region was identified on the $\mathrm{H} \alpha$ maps for each spectrum, to compute the metallicity gradient (see Sect. 4).

The spectral observation of the host of SN 1998bt indicates a redshift of $z=0.436$, much higher than the value assumed by Germany et al. (2004) and Pastorello et al. (2012). These authors assumed that the galaxy hosting SN 1998bt belonged to a cluster whose average redshift is $z=0.046$. Our spectrum shows that this galaxy is not a member of the cluster.

The new redshift was measured through the detection of strong emission lines $(\mathrm{H} \beta, \mathrm{H} \gamma,[\mathrm{O} \mathrm{III}] \lambda \lambda 5007,4959$ and [O II] 13727). This suggest that SN 1998bt was not a SN 1987A like event, but a much brighter object with $V_{\max }=-21.6 \mathrm{mag}$. There is no spectroscopic classification of SN 1998bt, so this event could have been a super-luminous SN (SLSN) with a light curve similar to SN 2010gx (Pastorello et al. 2010), which also has a slow rising and dome-like light curve. Moreover, the new redshift makes the host of SN 1998bt a galaxy with a normal $M_{B}=-18.58 \mathrm{mag}$ (here we applied a $k$-correction of +0.73 mag based on $m_{B}-m_{R}$, from http: //kcor . sai .msu . $\mathrm{ru}$ ) rather than a very faint galaxy with $M_{B} \approx-13$ mag. We also note that the metallicity measured with the $R 23$ method (see Sect. 4) for this host galaxy is $12+\log (\mathrm{O} / \mathrm{H})=7.83$ dex, which is consistent with the abundances of dwarf galaxies hosting SN 2010gx-like events (Chen et al. 2013). Therefore, SN 1998bt is no longer considered as part of our BSG SN sample.

\section{Indirect metallicity measurements}

It is well established that luminous galaxies have higher integrated or central metallicity than low-luminosity galaxies.

Tremonti et al. (2004) present a simple relation that, given the absolute $B$-band magnitude of the host, allows us to estimate the metallicity - the oxygen abundance $12+\log (\mathrm{O} / \mathrm{H})-$ at the galaxy center. The absolute $B$-band magnitude for each galaxy was computed from the apparent magnitude listed in the Asiago Supernova Catalogue (ASC, Barbon et al. 1999, where the magnitudes are mainly taken from de Vaucouleurs et al. 1991), and with distance moduli and Milky Way extinction as reported in Table 1. The distance was computed from the known redshift combined with the Hubble law, where we assumed $H_{0}=73 \mathrm{~km} \mathrm{~s}^{-1} \mathrm{Mpc}^{-1}$ (Spergel et al. 2007). For very 
Table 3. Metallicity estimates and normalised cumulative rank (NCR) pixel index for the sample of BSG SNe.

\begin{tabular}{|c|c|c|c|c|c|c|c|c|}
\hline $\mathrm{SN}$ & $\begin{array}{c}12+\log (\mathrm{O} / \mathrm{H}) \\
\text { (indirect } \\
\text { at the host center, } \\
\text { dex })\end{array}$ & $\begin{array}{c}12+\log (\mathrm{O} / \mathrm{H}) \\
(\text { indirect } \\
\left.\text { at } r_{\mathrm{SN}}, \operatorname{dex}\right)\end{array}$ & $\begin{array}{c}12+\log (\mathrm{O} / \mathrm{H}) \\
(\mathrm{N} 2 \text { at the } \\
\text { host center, dex })\end{array}$ & $\begin{array}{c}12+\log (\mathrm{O} / \mathrm{H}) \\
\left(\mathrm{N} 2 \text { at } r_{\mathrm{SN}}, \operatorname{dex}\right)\end{array}$ & $\begin{array}{l}12+\log (\mathrm{O} / \mathrm{H}) \\
(\mathrm{N} 2 \text { of the } \mathrm{H} \text { II } \\
\text { region closest } \\
\text { to the } \mathrm{SN}, \text { dex })\end{array}$ & $\begin{array}{c}\text { H II region-SN } \\
\text { distance } \\
(\mathrm{kpc})\end{array}$ & $\begin{array}{c}12+\log (\mathrm{O} / \mathrm{H}) \text { gradient } \\
\left(\operatorname{dex} R_{25}^{-1}\right)\end{array}$ & NCR \\
\hline 1909A & 9.13 & 8.30 & $8.79 \pm 0.06$ & $7.96 \pm 0.10$ & $8.23 \pm 0.20$ & 7.66 & $-0.900 \pm 0.085$ & 0 \\
\hline $1982 \mathrm{~F}$ & 8.85 & 8.83 & $8.24 \pm 0.09$ & $8.26 \pm 0.12$ & $8.21 \pm 0.20$ & 0.36 & $0.078 \pm 0.256$ & 0.113 \\
\hline 1987A & 8.57 & 8.45 & $8.46 \pm 0.06^{* *}$ & $8.37^{* * *}$ & - & - & $-0.323 \pm 0.215^{* *}$ & - \\
\hline $1998 \mathrm{~A}$ & 8.76 & 8.48 & $8.78 \pm 0.03$ & $8.68 \pm 0.06$ & $8.63 \pm 0.20$ & 0 & $-0.157 \pm 0.076$ & 0.608 \\
\hline $2000 \mathrm{cb}$ & 8.82 & 8.56 & $8.68 \pm 0.03$ & $8.45 \pm 0.06$ & $8.39 \pm 0.20$ & 1.24 & $-0.411 \pm 0.096$ & 0 \\
\hline NOOS...* & 8.94 & 8.50 & $9.02 \pm 0.13$ & $8.39 \pm 0.28$ & $8.57 \pm 0.20$ & 2.44 & $-0.677 \pm 0.250$ & 0.076 \\
\hline $2004 \mathrm{ek}$ & 8.94 & 8.59 & - & - & - & - & - & - \\
\hline $2004 \mathrm{em}$ & 8.90 & 8.55 & $8.72 \pm 0.05$ & $8.56 \pm 0.11$ & $8.59 \pm 0.20$ & 1.16 & $-0.213 \pm 0.122$ & 0.024 \\
\hline $2005 \mathrm{ci}$ & 8.63 & 8.42 & $8.49 \pm 0.02$ & $8.31 \pm 0.04$ & $8.31 \pm 0.20$ & 0 & $-0.412 \pm 0.089$ & 0.179 \\
\hline $2005 \mathrm{dp}$ & 8.70 & 8.40 & $8.49 \pm 0.03$ & $8.27 \pm 0.07$ & $8.37 \pm 0.20$ & 3.38 & $-0.336 \pm 0.094$ & 0.510 \\
\hline $2006 \mathrm{~V}$ & 9.16 & 8.72 & $8.81 \pm 0.05$ & $8.35 \pm 0.11$ & $8.36 \pm 0.20$ & 6.03 & $-0.487 \pm 0.101$ & 0 \\
\hline 2006au & 8.97 & 8.58 & $8.95 \pm 0.06$ & $8.49 \pm 0.12$ & $8.54 \pm 0.20$ & 0 & $-0.562 \pm 0.113$ & 0.073 \\
\hline 2009E & 8.48 & 8.21 & $8.44 \pm 0.05$ & $8.22 \pm 0.08$ & $8.22 \pm 0.20$ & 0 & $-0.391 \pm 0.112$ & 0.857 \\
\hline$\langle\mathrm{BSG} \mathrm{SNe}\rangle$ & $8.83 \pm 0.06$ & $8.51 \pm 0.04$ & $8.66 \pm 0.07$ & $8.36 \pm 0.05$ & $8.40 \pm 0.05$ & $2.02 \pm 0.80$ & $-0.399 \pm 0.072$ & $0.222 \pm 0.089$ \\
\hline
\end{tabular}

Notes. In the 2nd and 3rd columns we report the indirect metallicity estimates at the host center and at the SN distance. The direct metallicity measurements at the host center and at the SN distance are reported in the 4th and 5th columns. The quoted error is the fit uncertainty. The systematic error for the N2 method is 0.18 dex. Columns 6 and 7 include the oxygen abundance of the H II region closest to the SN position, and the distance in kpc between this H II region and the SN position. The last two columns show the measured host metallicity gradient and the NCR index for each SN. The mean value and the uncertainty of each quantity for the entire sample is reported in the last row of the table. ${ }^{(*)}$ Full name OGLE-2003-NOOS-005. ${ }^{(*)}$ From Pagel et al. (1978). ${ }^{(* *)}$ From Russell \& Dopita (1990); it is the average metallicity of the H II regions in the LMC.

nearby objects $(z<0.01)$ we adopted redshift independent distances from $\mathrm{NED}^{2}$. The extinction due to the dust along the line of sight in the Milky Way was corrected by using the data from Schlegel et al. (1998) ${ }^{3}$. Our sample includes a few (5) large galaxies having absolute magnitude similar or lower than that of the Milky Way $\left(M_{B}^{\mathrm{MW}}=-20.2\right)$. The faintest galaxy is characterized by $M_{B}=-17.5 \mathrm{mag}$. The central metallicities from $M_{B}$ are reported in the second column of Table 3.

At the same time, several authors (e.g. Pilyugin et al. 2004; Pilyugin \& Thuan 2007) have shown that H II regions at large distances from the center of their galaxy have lower metallicity than those close to the center. Therefore, a first and indirect approach (see e.g. Boissier \& Prantzos 2009) to infer the metallicity of 1987A-like $\mathrm{SNe}$ is to compute the central metallicity of the hosts from their absolute $B$-band magnitude and then to use the metallicity gradients in the literature to estimate the value at the SN distance from the nucleus.

The de-projected, normalized distance $\left(r_{\mathrm{SN}} / R_{25}\right)$ from the host galaxy center for each BSG SN was determined following Hakobyan et al. (2009). Given the SN and the host nucleus coordinates, the major $\left(2 R_{25}\right)$ and minor ( $\left.2 \mathrm{~b}\right)$ axes along with the position angle (PA) of the galaxy are the data needed to derive $r_{\mathrm{SN}} / R_{25}$. We also included a correction of the inclination based on the morphological type of the galaxy (t-type), following Hakobyan et al. (2012). Both the input data and the resulting $r_{\mathrm{SN}} / R_{25}$ are reported in Table 1 , and further details are given in the notes to that Table. The main sources for the galaxy properties were ASC, NED, and SIMBAD ${ }^{4}$. The normalized distances that we obtained are smaller than those reported by Pastorello et al. (2012, their Table 4). This is simply because they used galaxy radii from HyperLeda ${ }^{5}$, which are smaller than those reported by NED and/or by the ASC.

\footnotetext{
2 http://ned.ipac.caltech.edu

3 Extinction values are obtained through the NASA/IPACInfraredScienceArchive

4 http://simbad.u-strasbg.fr/simbad

5 http://leda.univ-lyon1.fr
}

By using the average gradient of the galaxies presented by Pilyugin et al. (2004) $\left(-0.47\right.$ dex $\left.R_{25}^{-1}\right)$, we then extrapolated the metallicity at the SN normalized and de-projected distance. This estimate is given in the third column in Table 3 . We note that for NGC 4490 and M 101 their measured gradients were used, which are published by Pilyugin \& Thuan (2007) and Kennicutt et al. (2003) ( $-0.063 \operatorname{dex} R_{25}^{-1}$ and $-0.90 \operatorname{dex} R_{25}^{-1}$, respectively). The choice of the average gradient from Pilyugin et al. (2004) is justified by the fact that gradients expressed in dex $R_{25}^{-1}$ exhibit a smaller range of values than those expressed in dex $\mathrm{kpc}^{-1}$, as noticed by Garnett et al. (1997).

Overall the indication that comes from the indirect measurements is that BSG SNe arise from marginally sub-solar metallicity environs $\left(\left\langle 12+\log (\mathrm{O} / \mathrm{H})_{\mathrm{BSG}}\right\rangle=8.51 \pm 0.04 \mathrm{dex}\right)$, as suggested by van den Bergh \& McClure (1989) and Pastorello et al. (2012).

The obtained values for the host galaxy $M_{B}$, for the SN-host distance and the metallicity at $r_{\mathrm{SN}}$ are more interesting when compared to those of different SN types. To make this comparison, we built large samples of galaxies that hosted Type II, IIP, IIn, IIb, Ib and Ic SNe using the ASC. These samples were built with the following criteria: a) all hosts at redshift $z>0.023$ were excluded, like in Kelly \& Kirshner (2012), to avoid a bias towards very bright SNe. In the sample of 13 BSG SNe that means the exclusion of OGLE-2003-NOOS-005 from the comparisons. b) We also excluded galaxies more inclined than $i>75 \mathrm{deg}$ to avoid large uncertainties in the de-projected distances. c) Finally, only those galaxies with known PA, major and minor axes, t-type and apparent $B$ magnitude were included.

For each host $M_{B}, r_{\mathrm{SN}} / R_{25}$ and the metallicity at the SN distance were computed as we did for our 1987A-like SNe, and for each type the cumulative distribution (CDF) of these quantities was plotted in Fig. 1. BSG SNe seem to be located in slightly fainter galaxies than those of other SN types (see left panel of Fig. 1). BSG SNe are also located more distant from the center than SNe II and IIP, and therefore also more distant than $\mathrm{SNe}$ Ibc. A similar trend is followed by the SN IIb population (see central panel of Fig. 1). Finally, BSG SNe present a lower 

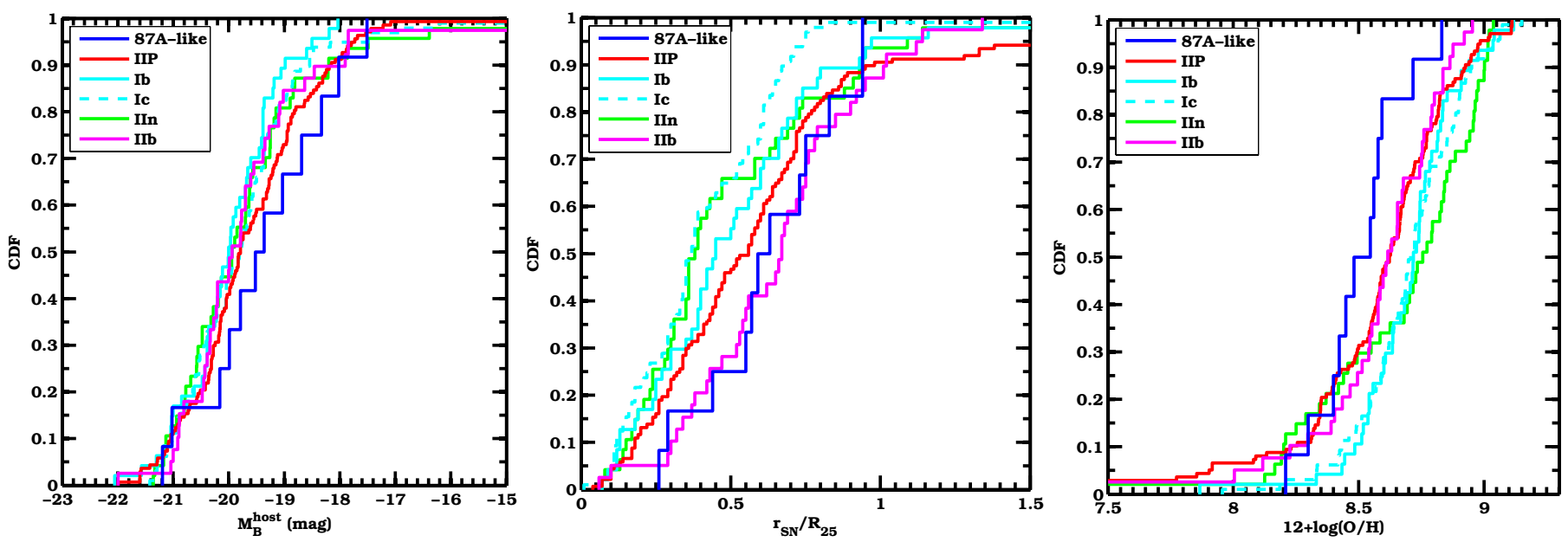

Fig. 1. Cumulative distributions of the host galaxy absolute $B$ magnitudes (left panel), of the SN-host center normalized offsets (central panel), and of the extrapolated metallicity at the SN distance (right panel), for our BSG SN sample and for other CC SN types. The metallicity has here been obtained by the indirect method outlined in Sect. 3 .

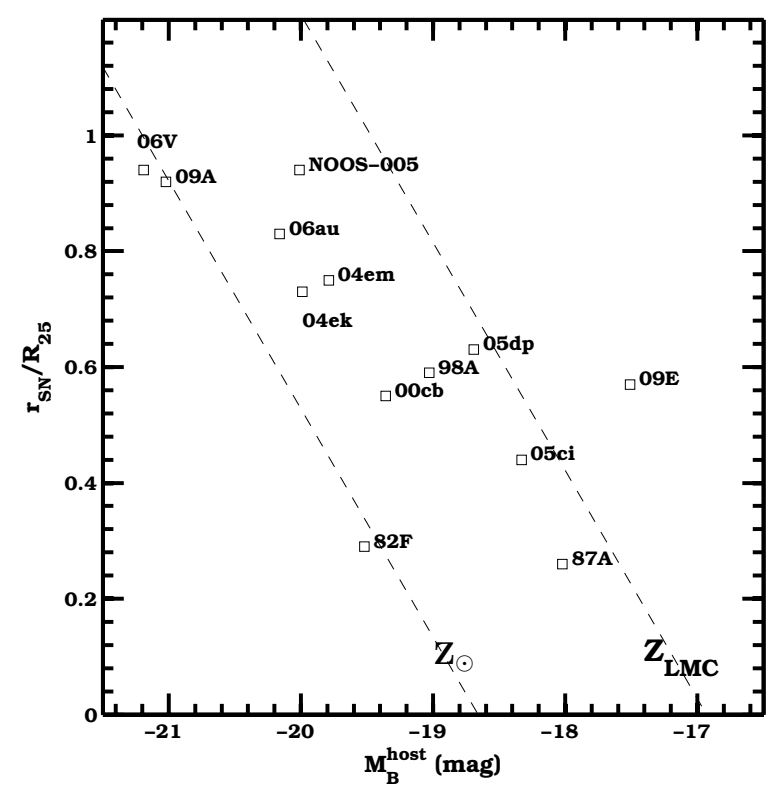

Fig. 2. Normalized SN-host center offset vs host galaxy absolute $B$ magnitude for each BSG SN in our sample. These SNe either exploded in low-luminous galaxies or in the outer parts of brighter hosts. Also shown are the iso-metallicity curves for $Z_{\odot}$ and $Z_{\mathrm{LMC}}$, where we assume the luminosity-metallicity relation and the mean gradient described in Sect. 3.

(indirect) metallicity at their positions (see right panel of Fig. 1). We note that our results concerning the other SN types (in particular the SN-host center distances) are consistent with those found by Kelly \& Kirshner (2012). The difference in metallicity between 1987A-like SNe and SNe IIP is not statistically significant (a Kolmogorov-Smirnov test, hereafter K-S, gives a $p$-value of 0.46). The difference in distance have higher statistical significance $(p$-value $=0.32)$. Obviously these differences are larger when we compare BSG SNe and SNe Ibc, which are known to be located even more centrally than SNe IIP (e.g. Hakobyan 2008).

Figure 2 illustrates that BSG SNe are at lower metallicity because either they are located in faint galaxies, which are intrinsically metal poor, or they are placed in the metal-poor outskirts of bright spiral galaxies. SN $1982 \mathrm{~F}$ is located quite close to the center of its bright host. However, in Sect. 4.2 we will see that this galaxy is in fact intrinsically metal-poor.

\section{Direct metallicity measurements}

We make use of the emission-line diagnostic named N2 (Pettini \& Pagel 2004) to obtain direct metallicity measurements of 11 BSG SN host galaxies. This diagnostic has been used in similar studies, for instance in Thöne et al. (2009), Leloudas et al. (2011), Anderson et al. (2010), Sanders et al. (2012) and Stoll et al. (2013).

$\mathrm{N} 2$ is the logarithm of the flux ratio between [N II] $\lambda 6584$ and $\mathrm{H} \alpha$. Since these two lines are close in wavelength, the method is neither affected by extinction effects nor by differential slit losses due to lack of an atmospheric dispersion corrector (ADC). We note that FORS2 is equipped with an ADC (Avila et al. 1997), whereas the ADC was not used when we observed with ALFOSC. The expression to obtain the oxygen abundance from $\mathrm{N} 2$ is $12+\log (\mathrm{O} / \mathrm{H})=9.37+2.03 \times \mathrm{N} 2+1.2 \times \mathrm{N} 2^{2}+$ $0.32 \times \mathrm{N} 2^{3}$ (Pettini \& Pagel 2004). The systematic N2 uncertainty on $12+\log (\mathrm{O} / \mathrm{H})$ is $0.18 \mathrm{dex}$ and dominates the overall error that affects the flux measurements. Therefore, we assume a total uncertainty of 0.2 dex on the single metallicity measurement. The same choice was done by Thöne et al. (2009), who used the same observational setup.

The [N II] $\lambda 6584$ and $\mathrm{H} \alpha$ fluxes were determined in the following way: a) we carefully fit the continuum around the lines. We notice that, despite the careful fit of the continuum, the underlying $\mathrm{H} \alpha$ stellar absorption might affect the result, pushing the metallicity to higher values; however, the N2 measurements of three host galaxy nuclei obtained through the SDSS line fluxes (see Sects. 4.7, 4.8 and 4.11), which were measured after subtracting the stellar absorption (Bolton et al. 2012), do not differ significantly from our measurements at the same positions. b) Then, three Gaussians with a single FWHM (determined by the spectral resolution) and fixed wavelength centroids (known from the atomic physics) were simultaneously fit to [N II] $\lambda 6584$, [N II] $\lambda 6548$ and $\mathrm{H} \alpha$. Since [N II] $\lambda 6548$ is difficult to fit given its faintness, its flux was fixed to be $1 / 3$ of [N II] $\lambda 6584$ (see Osterbrock 1989). The fit of this faint and blended line is done to optimize the measurement of the $\mathrm{H} \alpha$ flux and remove possible contaminations, especially for the NOT spectra, which are of lower resolution. This procedure allowed us to measure the two important lines in a robust way. In Fig. 3 we show the spectrum of a bright and metal-poor H II region in UGC 6510 (top panel), and the triple-Gaussian fit that was performed on its $\mathrm{H} \alpha$ 

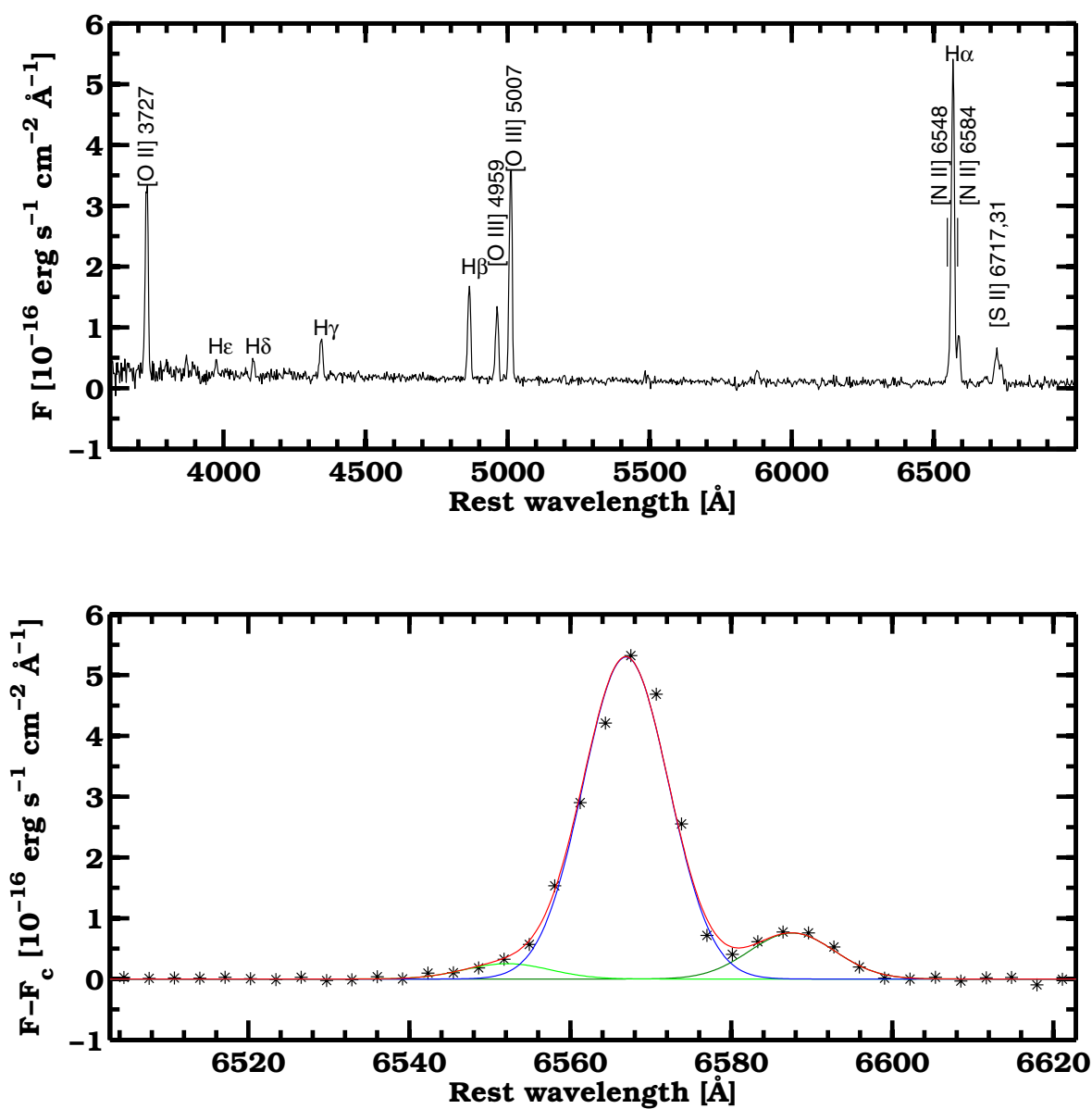

Fig. 3. (Top panel) An example of a bright H II region spectrum (in UGC 6510), where we identify the strongest detected lines. (Bottom panel) The continuum subtracted $\mathrm{H} \alpha$ and $[\mathrm{N}$ II] lines are shown along with the multiple Gaussian fit that allows us to deblend the three different features and to measure their fluxes. In red we indicate the total fit. In bright green, dark green and blue we show the single components fitting [N II] $\lambda 6548$, [N II] 26584 , and $\mathrm{H} \alpha$, respectively. The difference in wavelength between the centroids of each line are fixed, a single FWHM is used and the [N II] $\lambda 6584$ to [N II] $\lambda 6548$ flux ratio is fixed to 3.

and [N II] lines (bottom panel). In the case of SN 2005dp, the host galaxy was observed with ALFOSC using both grism \#4 and grism \#8 (which has a higher resolution), to compare the line ratios obtained at low (with the line deblending) and high resolution. We basically obtained the same line ratios, with an average difference smaller than $1 \%$.

For each H II region falling into the slit, the metallicity was measured with the N2 method. These values are reported on the continuum-subtracted $\mathrm{H} \alpha$ images at the position of each of these regions using a color map (see the top or top-right panels of Figs. 4-14). The width of each colored patch corresponds to the actual width of the spectral extraction. In the continuumsubtracted $\mathrm{H} \alpha$ images we also show the slit aperture and the 25th $B$-band magnitude contour, from the values reported in Table 1, as well as the image orientation and its scale in arcseconds. The center of the galaxy is indicated with a circle and the SN position with a star.

The flux at the $\mathrm{H} \alpha$ rest wavelength versus the distance in arcseconds from the SN position (or nucleus position) is shown in the top-left or central panels of Figs. 5-14. Here, we also report the metallicity values at their positions. The spatial uncertainty of each metallicity value corresponds again to the width of the spectral extraction.

Using the same formulae that were employed to de-project the distance of each SN from its host center, we compute the deprojected distance of each $\mathrm{H}$ II region with measured metallicity from the galaxy nucleus. The metallicity values versus these deprojected distances (normalized by the galaxy radius) are shown in the bottom panels of Figs. 4-14. Fitting a line to these points (we performed a chi-square fit), the metallicity was found to decrease as the distance from the center increases (with the exception of NGG 4490, which is an interacting galaxy). These line fits were used to interpolate the metallicity at the exact SN position (the SN distance is marked by a dotted, vertical line) when the $\mathrm{SN}$ does not fall onto a bright $\mathrm{H}$ II region. We found that the interpolated or extrapolated metallicity always matches within the error with the directly measured metallicity when the $\mathrm{SN}$ coincides in position with a bright $\mathrm{H}$ II region (see the 5th and 6th columns of Table 3). Therefore, for each SN it was assumed that the value from the linear fit is the best estimate of the $\mathrm{N} 2$ metallicity. These values are marked by a red, void square in the bottom panels of Figs. 4-14 and summarized in the 5th column of Table 3 . The uncertainty on the extrapolated value corresponds to the standard deviation of 1000 extrapolations from linear fits that were Monte Carlo simulated based on the uncertainty on the slope and on the central metallicity. These uncertainties do not consider the systematic error included in the N2 method. They only take into account the scatter of each metallicity estimate from the best linear fit, since for each estimate we observed a small scatter around the linear fit (see the bottom panels of Figs. 4-14) compared to the systematic 0.18 dex reported by Pettini \& Pagel (2004). We also note 
that our measurements involve a range of oxygen abundances $(12+\log (\mathrm{O} / \mathrm{H}) \approx 8.2-8.7 \mathrm{dex})$ that is limited compared to that investigated by Pettini \& Pagel (2004). In this interval, the scatter of the N2 relation is indeed smaller than $0.18 \mathrm{dex}$, as visible in their Fig. 1.

The strong-line diagnostic method that has been employed is not the only possibility. Other methods that are often used in the literature are the so called $\mathrm{O} 3 \mathrm{~N} 2$ and $\mathrm{R} 23 . \mathrm{O} 3 \mathrm{~N} 2$ is defined as $\mathrm{O} 3 \mathrm{~N} 2=\log [([\mathrm{O} \mathrm{III}] \lambda 5007 / \mathrm{H} \beta) /([\mathrm{N} \mathrm{II}] \lambda 6584 / \mathrm{H} \alpha)]$ and it is linked to the oxygen abundances by the following relation: $12+\log (\mathrm{O} / \mathrm{H})=8.73-0.32$ O3N2 (Pettini \& Pagel 2004). Like N2, it is based on ratios of lines that are close in wavelength. However, it is not always possible to detect all the four needed lines, and especially [O III] $\lambda 5007$ is faint at low metallicity. $\mathrm{R} 23$ is a method based on oxygen lines and $\mathrm{H} \beta(R 23=$ ([O II] $\lambda 3727+[\mathrm{O}$ III] $\lambda 4959+[\mathrm{O}$ III $] \lambda 5007) / \mathrm{H} \beta$ ). It is sensitive to the extinction, that it is usually estimated from the Balmer decrement (Osterbrock 1989), and to differential slit losses. The parameter $R 23$ is related to the metallicity by a polynomial relation given by e.g. Nagao et al. (2006). Also in this case, it is not always possible to detect all the lines that are needed to measure the $R 23$ parameter. In the case of SN 2005ci (see Fig. 10, center-left panel), we show the metallicities obtained with N2, $\mathrm{O} 3 \mathrm{~N} 2$ and $R 23$ for each $\mathrm{H}$ II region. We also present the values of $E(B-V)$ as estimated from the Balmer decrement, which are needed to compute the $R 23$ parameter. The $R 23$ estimates differ from those obtained with the other two methods by $\lesssim 0.1$ dex. This is not surprising, since there are offsets between the methods which are discussed in the literature (e.g. Kewley \& Ellison 2008).

In summary, we chose the N2 method for the following reasons: I) it is easier to observe the lines needed for this method than those needed for the other methods. II) N2 is less affected by extinction and differential slit losses. III) N2 has often been used in the literature to measure the metallicity of other $\mathrm{SN}$ types. Therefore, choosing N2 for our sample allows us to directly compare our results to those of other SN classes (see Sect. 4.12). IV) N2 is calibrated against metallicity measured with the electron temperature method (see Pettini \& Pagel 2004). This method is found to match the metallicities measured by the modeling of stellar spectra better than other methods (Bresolin et al. 2009). Therefore, since the solar metallicity $\left(Z_{\odot}\right)$ estimate is also based on spectral modeling (Asplund et al. 2009), N2 is appropriate to compare our results to $Z_{\odot}$.

In the following sections, each host is individually discussed together with its metallicity measurements.

\subsection{M $101-S N 1909 A$}

M 101 is a nearby spiral galaxy that covers several square arcminutes on the northern sky. SN 1909A exploded in the outskirts of this galaxy, far from any bright H II region. Since the metallicity gradient of this galaxy is well studied and presented by Kennicutt et al. (2003), we simply tested our N2 measurement on a bright $\mathrm{H}$ II region relatively close (de-projected distance of $7.66 \mathrm{kpc}$ ) to the position of SN 1909A (top panel of Fig. 4) against the published metallicity gradient (bottom panel of Fig. 4). We found a good match and therefore we proceeded with the extrapolation of the $12+\log (\mathrm{O} / \mathrm{H})$ to the $\mathrm{SN}$ distance, obtaining the lowest value of our sample $(12+\log (\mathrm{O} / \mathrm{H})=$ $7.96 \pm 0.10 \mathrm{dex})$. SN 1909A is thus a typical example of a BSG SN that exploded far from the metal-rich nucleus of a bright galaxy. The explosion site of the $\mathrm{SN}$ has a low oxygen abundance due to the metallicity gradient in the host.

\subsection{NGC $4490-S N 1982 F$}

NGC 4490 (see Fig. 5) is a nearby, interacting galaxy that hosted SN 1982F and also the Type IIb SN 2008ax (Taubenberger et al. 2011). Pilyugin \& Thuan (2007) published some metallicity measurements that we have complemented with our N2 estimates. In this case we placed the slit through a small $\mathrm{H}$ II region close to the location of SN $1982 \mathrm{~F}$ and through a bright $\mathrm{H}$ II region closer to the center. All the obtained $12+\log (\mathrm{O} / \mathrm{H})$ values fall below solar metallicity (consistently with the results by Pilyugin \& Thuan 2007), implying an extrapolated SN oxygen abundance of 8.2-8.3 dex (similar to that found in the LMC). The metallicity gradient is found to be unusually flat, likely because this galaxy is interacting with another one (NGC 4485). Perez et al. (2011) show that the central metallicity of interacting galaxies is diluted by tidally induced low-metallicity gas inflows, resulting in a flatter oxygen abundance gradient. We notice that using O3N2, Kelly \& Kirshner (2012) found $12+\log (\mathrm{O} / \mathrm{H})=$ 8.21 dex for SN 2008ax, confirming that NGC 4490 produces core-collapse (CC) SNe at relatively low metallicity. SN 1982F is an example of a BSG SN that is located not too far $\left(r_{\mathrm{SN}} / R_{25}=\right.$ 0.30 ) from the center of a galaxy that has an intrinsically low metallicity.

\subsection{IC $2627-S N 1998 A$}

IC 2627 is a southern object, characterized by strong star formation, as shown by the bright $\mathrm{H} \alpha$ knots that are mostly distributed along two arms. SN 1998A exploded in the outer region of one of these arms and was located on a bright $\mathrm{H}$ II region (see Fig. 6, top panel). This galaxy also hosted the normal Type II SN 1994R, whose position we marked with a diamond. The galaxy was observed along the two directions defined by the host center and the two SNe (see the slits marked with VLT 98A and VLT 94R in Fig. 6, top panel). Furthermore, we used two spectra from the ESO archive that included the flux of SN 1998 ${ }^{6}$. By reconstructing the slit positions of these two spectra, they are found to be placed almost through the galaxy center (see the slit marked NTT 1 in Fig. 6, top panel), and on the bright arm that hosted SN 1998A (slit marked NTT 2 in Fig. 6, top panel). The three different metallicity measurements at the exact position of SN 1998A converge to 8.62-8.68 dex, which is the highest value in our sample, consistent with solar abundance (8.69 \pm 0.05 dex; Asplund et al. 2009). The other measurements across the galaxy are all solar or super-solar, and overall the galaxy exhibits a very shallow metallicity gradient (Fig. 6, bottom panel). The region between SN 1994R and the host center is that with the highest N2 metallicity, close to 9.0 dex (Fig. 6, center panels). SN 1998A represents the rare counterexample of SN 1909A, since it is located in a solar-metallicity environment despite its relatively large distance $\left(r_{\mathrm{SN}} / R_{25}=0.59\right)$ from the nucleus.

\subsection{IC $1158-S N 2000 c b$}

IC 1158 is another spiral galaxy with diffuse star formation, particularly concentrated in its southern and central part (see Fig. 7, top-right panel). The slit, which was placed on the SN explosion site and through the galaxy nucleus, reveals that there is no significant $\mathrm{H} \alpha$ emission at the SN position. However, two bright knots are located a few arcseconds (the nearest at a de-projected distance of $1.24 \mathrm{kpc}$ ) from it and a total of $8 \mathrm{H} \mathrm{II} \mathrm{regions} \mathrm{fell}$ into the slit (see Fig. 7, top-left panel). This allowed us to map

6 From ESO program 60.D-0281, PI: J. Spyromilio. 
the metallicity gradient, as shown in the bottom panel of Fig. 7 and to estimate the oxygen abundance at the SN position to be about 8.5 dex. This is consistent with the result of Anderson et al. (2010).

\subsection{MASX J05553978-6855381 - OGLE-2003-NOOS-005}

2MASX J05553978-6855381 is a relatively distant galaxy $(z=0.03)$, which hosted OGLE-2003-NOOS-005. This SN exploded very far from the host center $\left(r_{\mathrm{SN}} / R_{25}=0.94\right)$ and not on a $\mathrm{H}$ II region, although close $(2.44 \mathrm{kpc})$ to a bright one (top panels of Fig. 8). For this galaxy, we measured the extent of the axes directly on the $B$-band image that we obtained at the VLT. The N2 measurements reveal a super-solar central metallicity and a gradient that seems to flatten in the outer part of the galaxy (bottom panel of Fig. 8). We note that the $\mathrm{H} \alpha$ line measurements at the exact host center is made difficult by significant stellar absorption. At the SN distance, the oxygen abundance is moderately sub-solar $(\sim 8.4 \mathrm{dex})$.

\subsection{IC $1303-S N 2004 e m$}

The spiral galaxy IC 1303 is placed in a region of the sky crowded with foreground stars. SN 2004em exploded in its outer part, close $(1.16 \mathrm{kpc}$ ) to a relatively bright $\mathrm{H}$ II region (see top panels of Fig. 9). We measured the metallicity at the host center and at four other positions, revealing a super-solar central metallicity and a shallow gradient (see bottom panel of Fig. 9). At the SN distance, the oxygen abundance is $12+\log (\mathrm{O} / \mathrm{H})=8.56 \mathrm{dex}$, which is consistent with solar metallicity within the errors.

\subsection{NGC $5682-S N 2005 c i$}

NGC 5682 is a strongly tilted ( $i=70 \mathrm{deg}$ ) galaxy, with two arms characterized by significant star formation. SN 2005ci is located quite close to the galaxy center $\left(r_{\mathrm{SN}} / R_{25}=0.44\right)$, on a bright $\mathrm{H}$ II region (top panel of Fig. 10). We placed one slit along the $\mathrm{SN}$-host center direction, and one along the major axis (see top panel of Fig. 10) The eight metallicity measurements that we obtained with two slits (see central panels of Fig. 10) show a steep gradient and a LMC-like metallicity at the SN position (bottom panel of Fig. 10). We have also included the N2 measurement at the center of the host from SDSS, finding a perfect match to our results. As explained in Sect. 4, for the H II regions that have fallen into the slit at the SN position we also measured the $\mathrm{O} 3 \mathrm{~N} 2$ and $R 23$ metallicities, which are presented in the bottom and center-left panels.

\subsection{NGC $5630-S N 2005 d p$}

NGC 5630 is a strongly tilted ( $i=72 \mathrm{deg}$ ) galaxy at low redshift $(z=0.008)$. It shows star formation mainly in the central region and in the eastern part. The slit was first placed (Fig. 11, central-left panel) at the SN position and on a reference star in the north of the galaxy. The second spectrum (Fig. 11, centralright panel) was taken along the major axis of the galaxy, as shown in Fig. 11 (top panel). A total of 16 metallicity measurements were obtained, revealing slightly sub-solar metallicity at the center and a lower, LMC metallicity in the outer parts. The $\mathrm{SN}$, which is located at $r_{\mathrm{SN}} / R_{25}=0.63$, exploded in an environment with $12+\log (\mathrm{O} / \mathrm{H})=8.27 \pm 0.07 \mathrm{dex}$ (Fig. 11, bottom panel). Our central measurements are perfectly consistent with the N2 measurement from the SDSS spectrum, which is reported in the bottom panel of Fig. 11.

\subsection{UGC $6510-S N 2006 \mathrm{~V}$}

UGC 6510 is a bright, face-on galaxy that is formed by multiple arms and characterized by a significant $\mathrm{H} \alpha$ luminosity. SN 2006V occurred in the outermost part of the galaxy and not on a detectable $\mathrm{H}$ II region. We observed this host with both the VLT and the NOT. The NOT-ALFOSC slit was placed in order to catch the flux of the brightest H II region close to the SN position (see the top and center-left panels of Fig. 12). The VLT-FORS2 slit was positioned at the exact SN position and through the host center (see the top and center-right panels of Fig. 12). Metallicities obtained with the VLT and with the NOT at similar deprojected distances do match very well, confirming that the deblending of $\mathrm{H} \alpha$ and [N II] lines (necessary for the NOT spectra) gives correct values for their fluxes. A total of $14 \mathrm{H}$ II regions were investigated, allowing for a precise determination of the metallicity gradient (see the bottom panel of Fig. 12). The extrapolated metallicity at the distance of SN $2006 \mathrm{~V}$ is $12+\log (\mathrm{O} / \mathrm{H})=8.35$ dex.

\subsection{UGC $11057-S N 2006 a u$}

UGC 11057 is a spiral galaxy that appears strongly tilted ( $i=$ $66 \mathrm{deg}$ ) on the sky (see the top-right panel of Fig. 13). The brightest $\mathrm{H} \alpha$ luminosity comes from the outer part of the arms that form this host. A bright, saturated foreground star affected the quality of the continuum-subtracted $\mathrm{H} \alpha$ image, leaving some residuals that we manually removed. SN 2006au exploded in spatial coincidence with a bright and extended $\mathrm{HII}$ region (see the top panels of Fig. 13), onto which we placed the slit, at $r_{\mathrm{SN}} / R_{25}=0.83$. A total of eight metallicity measurements were obtained and from the computed gradient we obtained $12+\log (\mathrm{O} / \mathrm{H})=8.49 \mathrm{dex}$ at the $\mathrm{SN}$ position, consistent with the single-measurement value at the exact SN position (see the bottom panel of Fig. 13). Similar to the host of OGLE-2003NOOS-005, UGC 11057 shows a gradient that tends to flatten in the outer regions.

\subsection{NGC $4141-$ SN $2009 E$}

NGC 4141 is the barred spiral galaxy that hosted SN 2009E. It was observed with the NOT, by placing the slit through the SN position and through a very bright $\mathrm{H}$ II region closer to the host center (see the top panels of Fig. 14). A total of five metallicity measurements were obtained, including that at the exact $\mathrm{SN}$ position. The galaxy has a moderately sub-solar central metallicity (as also found by SDSS) that further decreases with the distance from the center (see the bottom panel of Fig. 14). At the $\mathrm{SN}$ position the value is lower than the LMC metallicity.

\subsection{Metallicity results for the SN 1987A-like sample and comparisons to other SN types}

The average metallicity that we obtain from the direct measurements for our sample is $\left\langle 12+\log (\mathrm{O} / \mathrm{H})_{\mathrm{BSG}}\right\rangle=8.36 \pm 0.05 \mathrm{dex}$ (where the uncertainty is given by $\sigma / \sqrt{N}$ and $N=12$ ). This value is moderately sub-solar and compatible with that of the LMC, and therefore similar to that of SN 1987A. However, we also find SN 1987A-like transients at solar or almost solar metallicity, like SNe 1998A and 2004am. 


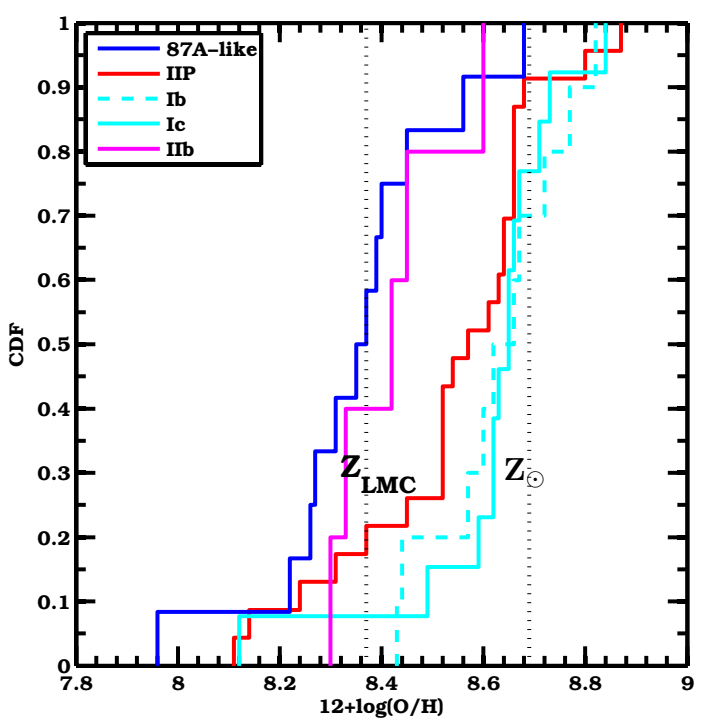

Fig. 15. Cumulative distribution of the directly measured (N2 method) metallicity of our BSG SNe and of samples of SNe IIP, Ib, Ic from Anderson et al. (2010) and of SNe IIb from Sanders et al. (2012). BSG SNe tend to explode at LMC metallicity $(12+\log (\mathrm{O} / \mathrm{H})=$ 8.3-8.4 dex). However, a few objects are found at almost solar metallicity $(12+\log (\mathrm{O} / \mathrm{H})>8.5$ dex $)$. BSG SNe clearly have lower metallicity than SNe IIP.

It is of interest to compare the metallicity of our sample to those of other subtypes. To make a proper comparison, we use the data from Anderson et al. (2010) and Sanders et al. (2012) that were obtained with the N2 method. From Anderson et al. (2010), in order to prevent gradient effects, we selected only those SNe IIP, Ib and Ic that are closer than $3 \mathrm{kpc}$ from the inspected H II region. SN 2000cb was also excluded from the sample of SNe IIP. From Sanders et al. (2012) we selected only the $\mathrm{SNe}$ IIb which have a H II region spectrum at the exact $\mathrm{SN}$ position. We computed the cumulative distributions of the metallicity for our sample (12 objects) and for the selected SNe IIP (23 objects), for the $\mathrm{SNe} \mathrm{Ib}$ (10 objects), for the SNe Ic (13 objects) and for the SNe IIb (5 objects). These are presented in Fig. 15. It is evident from the comparison that the population of 1987Alike $\mathrm{SNe}$ is associated with lower metallicity environs than those of normal SNe IIP $\left(\left\langle 12+\log (\mathrm{O} / \mathrm{H})_{\text {IIP }}\right\rangle=8.54 \pm 0.04 \mathrm{dex}\right)$. A $\mathrm{K}-\mathrm{S}$ test between 1987A-like and IIP events gives a $p$-value of 0.006 , revealing that this difference is statistically significant. $\mathrm{SNe} \mathrm{Ib}$ and Ic are at even higher metallicities than SNe IIP, as already shown by Anderson et al. (2010) and Kelly \& Kirshner (2012). Interestingly, SNe IIb were found to have a metallicity distribution that is very similar $\left(\left\langle 12+\log (\mathrm{O} / \mathrm{H})_{\mathrm{IIb}}\right\rangle=8.42 \pm\right.$ 0.05 dex) to those of our BSG SNe, as suggested by the fact that they are also preferentially located in the outer regions of their hosts (see Fig. 1, central panel). However, the sample of $\mathrm{SNe} \mathrm{IIb}$ is rather small. We note that the systematic uncertainties on the single measurements were not taken into account when we determined the statistically significant difference between BSG SNe and SNe IIP. To estimate the uncertainty on the $p$-value, $N=10000$ Monte Carlo simulations of the metallicity measurements were made for our BSG SNe and for the SNe IIP, based on the fit errors given in Table 3 (Col. 5) for our BSG SNe and assuming 0.1 dex uncertainty for the SNe IIP, which is the average uncertainty for our sample. For each couple of simulations we computed the K-S test obtaining $\mathrm{N} p$-values. Then, the cumulative distribution of these $\mathrm{N} p$-values was built and $68 \%$ of them were found to be lower than 0.033 . This can

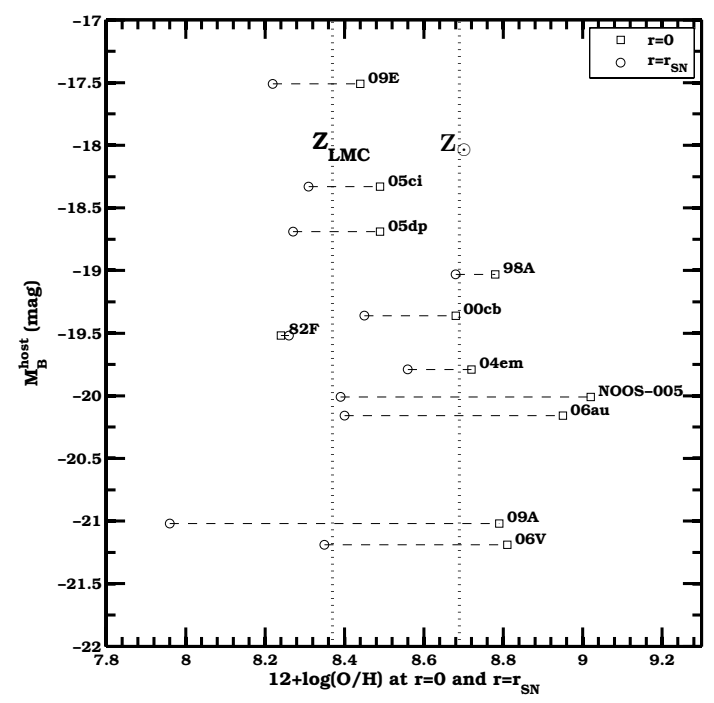

Fig. 16. Directly measured metallicity at the host center and at the SN distance vs. host galaxy $M_{B}$. A low central metallicity has been found for low-luminous galaxies, a higher metallicity for the luminous ones. This is consistent with previous studies concerning the luminosity-metallicity relation. The metallicity gradient explains why also the $\mathrm{SNe}$ hosted by bright galaxies were found at sub-solar metallicity.

be taken to represent the error on the $p$-value and would confirm that the difference between BSG SNe and SNe IIP is statistically significant $(>2 \sigma$ level). On the other hand, if we assume the typical 0.2 dex error for each N2 metallicity estimate, $68 \%$ of the $p$-values sits below 0.189 , and only $35 \%$ of them are found below 0.05 . However, as outlined in Sect. 4 , we think that 0.2 dex could be an overestimate of the actual uncertainty on the SN metallicity. This is supported by the fact that we are investigating a narrow metallicity range where the dispersion in the Pettini \& Pagel (2004) relation is smaller than $0.18 \mathrm{dex}$ and also by the observed dispersion around the metallicity linear fit (bottom panels of Figs. 4-14), which is clearly smaller than 0.18 dex for most of the galaxies.

The metallicity gradient of our galaxy sample (see the last column of Table 3 ) has an average of $-0.399 \pm 0.072$ dex $R_{25}^{-1}$. The host galaxies of SNe 1982F, 1998A, and 2004em show a much flatter behavior, whereas M 101 and the host of OGLE2003-NOOS-005 exhibit a steeper slope. The average gradient that we found is very similar to that found by Pilyugin et al. (2004) for their larger sample of galaxies.

The measured central metallicity (see the 4th column of Table 3) is higher for the bright galaxies than for the faint ones (with the exception of the interacting galaxy NGC 4490), as expected by previous studies (e.g. Tremonti et al. 2004). We plot $M_{B}$ versus central metallicity from N2 in Fig. 16. In this figure we also plot the N2 metallicity at the SN position, showing the effect of the metallicity gradient that is particularly important for those $\mathrm{SNe}$ that were hosted by bright, metal rich galaxies.

A comparison between indirect and direct measurements at the host center reveals a general agreement within $\sim 0.2$ dex, with the exception of NGC 4490 (host of SN 1982F) that is bright but intrinsically metal poor. We note that the metallicity-luminosity relation that we use (Tremonti et al. 2004) is known to be affected by a large scatter. When we compare indirect and direct measurements at the SN position, we found a good correspondence within $\sim 0.2$ dex for the majority of the objects. The gradient that we used to extrapolate the indirectly-measured metallicity at the $\mathrm{SN}$ distance is a good approximation for most of 


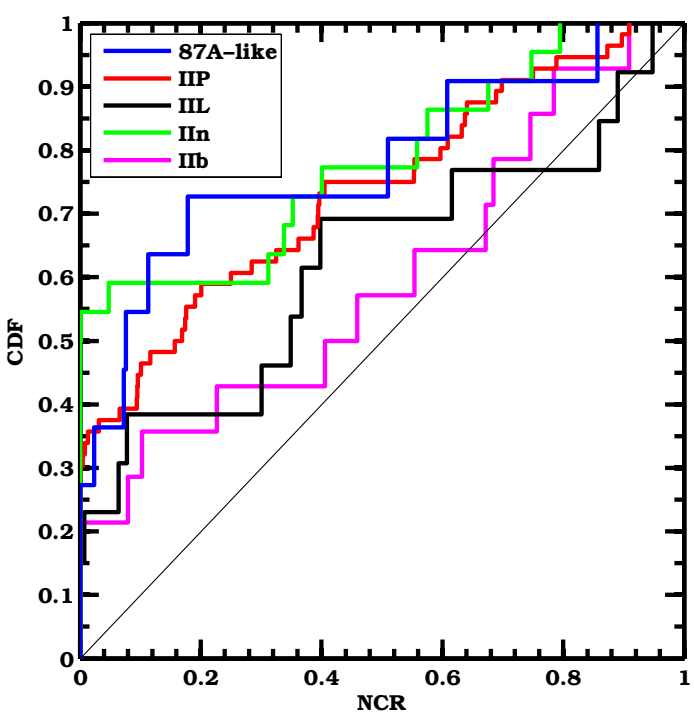

Fig. 17. Cumulative distribution of the NCR values for our BSG SNe and for samples of different CC SN types from Anderson et al. (2012). BSG SNe seem to have a degree of association to star-forming regions similar to that of SNe IIP and IIn.

the hosts (it is close to our directly-measured average gradient). However, some hosts have a shallower or steeper gradient, and this contributes to the discrepancies found for some events. Overall, we confirm that the indirect approach to measure the metallicity is useful to suggest possible differences among SN samples. However, it is necessary to directly measure the metallicity through strong-line diagnostics to obtain robust results for individual objects.

\section{Association with star-forming regions}

The spectral observations of the BSG SN host galaxies revealed that some of our objects are not spatially coincident with bright $\mathrm{H}$ II regions. Therefore, armed with the continuum-subtracted $\mathrm{H} \alpha$ images, we proceeded to quantify the spatial association of our $\mathrm{SNe}$ (a total of 11 hosts were observed in $R$ and $\mathrm{H} \alpha$ ) to starforming regions, which are $\mathrm{H} \alpha$ emitters. We made use of the normalised cumulative rank pixel function (NCR), first presented by James \& Anderson (2006) and then used by Anderson \& James (2009) and Anderson et al. (2012) to compare the association of different CC SN types to H II regions. This method consists of the following steps: a) each pixel in the continuum-subtracted $\mathrm{H} \alpha$ image is ranked according to its flux value; b) then, the cumulative distribution of the pixel values is computed; c) the value of this cumulative distribution at the rank value of the SN pixel corresponds to the NCR value for the SN. The NCR is 0 if the $\mathrm{SN}$ is not associated to $\mathrm{H}$ II regions, and 1 if the $\mathrm{SN}$ falls onto the brightest pixel. Following James \& Anderson (2006) and Anderson et al. (2012), the NCR analysis was performed on the $3 \times 3$ rebinned continuum-subtracted $\mathrm{H} \alpha$ image, in order to avoid possible errors in the determination of the SN position. The pixels that we used for the statistic were only those located within the 25 th $B$ magnitude iso-contour. We note that internal extinction is not accounted for in the NCR analysis.

The NCR values that we obtained are reported in the last column of Table 3 and compared to those obtained by Anderson et al. (2012) for other types of CC SNe in Fig. 17. We notice that the NCR of both SN 2005dp and SN 1982F were already computed by Anderson \& James (2009) and Anderson et al. (2012).
For these two $\mathrm{SNe}$, we obtained values which are very close to theirs (within 0.08 and 0.02 respectively).

Interestingly, the cumulative distribution of our NCR values follows that of SNe IIP and IIn, indicating a moderate association to star-forming regions for our 1987A-like SNe.

\section{Discussion}

This study is based mainly on local metallicity measurements. We have shown that it is important to take the metallicity gradient into account, but also remark that, even though our measurements are made at the exact SN position, the size of the $\mathrm{HII}$ regions that we inspected is always on the order of $\sim 0.1-1 \mathrm{kpc}$. It is possible that any particular $\mathrm{SN}$ sits in a small pocket of lower (or higher) metallicity within the larger H II region. Some apparent associations between SN position and $\mathrm{H}$ II region may also be due to projection effects.

By observing more targets, we reduce the impact of potential local inhomogeneities in the metallicity estimates and the results obtained are valid in a statistical sense. However, it is worth noting that we do not find any strong metallicity difference among $\mathrm{H}$ II regions that sit close to each other, and that there are no large deviations from the metallicity gradients that we measure. This suggests that the metallicity varies smoothly across the galaxy and that the presence of significant inhomogeneities is likely to be rare.

An important result from our study is that, on average, the BSG SN population is located at lower metallicity than that of normal SNe IIP arising from RSGs. There may be biases in the samples of BSG SNe and SNe IIP that we use for the comparison and in the following we discuss them.

Most of the BSG SNe were discovered by amateur astronomers or by targeted surveys (Pastorello et al. 2012), which preferentially observe bright, and therefore metal-rich galaxies. However, this is also the case for the SNe IIP that were taken from Anderson et al. (2012). This effect might bias both samples towards higher metallicities. However, we still see a difference in metallicity between the two populations.

The vast majority of our hosts and of those observed by Anderson et al. (2012) are nearby galaxies (recessional velocity $v<6000 \mathrm{~km} \mathrm{~s}^{-1}$ ), and this distance cut reduces the bias of the two samples towards intrinsically very bright objects. However, the light curves of SNe IIP exhibit a long plateau phase (3-4 months), whereas the peak of SN 1987A-like events lasts a few weeks. This could bias the SN 1987A-like population that might contain a higher fraction of faint, undetected objects. In fact, many of the discovered BSG SNe are brighter (Pastorello et al. 2012) than their prototype, SN 1987A. The average peak brightness of BSG SNe is $\left\langle M_{R}^{\max }\right\rangle \approx-17$ (data from Pastorello et al. 2012 and Arcavi et al. 2012), which does not differ considerably from that of SNe IIP (Kelly \& Kirshner 2012 indicate $\left\langle M_{R}^{\max }\right\rangle \approx-16$ for the Type II SN class). We looked for possible correlations between the metallicity of BSG SNe and their light-curve properties. It was found that the peak brightness of our transients (from Pastorello et al. 2012) does not correlate with the measured metallicity. This is also true for the light-curve shape. For example, SN 2000cb is at almost solar metallicity and SN 2005ci is clearly at LMC metallicity, but their light-curve shapes are very similar (Kleiser et al. 2011; Arcavi et al. 2012).

In summary, BSG SNe are - on average - at lower metallicity compared to those of SNe IIP and Ibc and the moderately low metallicity is a characteristic of the majority of BSG SNe. Although it is clear from stellar evolution theory that also other 
effects can influence the star - such as rotation, mixing and binarity - our simple observational test indicates that metallicity likely plays a role in the formation of exploding BSGs, at least for a large fraction of them. This would find a natural explanation if the progenitor was a single star. According to single-star evolution models, LMC metallicity can induce a $\sim 20 M_{\odot}$ star to explode in the blue part of the HR diagram (Brunish \& Truran 1982).

However, our results also indicate that two SN 1987Alike SNe exploded at near solar metallicity (SN 2004em and SN 1998A), and it is also clear that even if the mean metallicity is low, it is not very low. This supports the idea that, at least for some BSG SNe, a moderately low metallicity is not a necessary ingredient. We note that theoretical models show that a star in a binary system (Podsiadlowski 1992) or a single, fast rotating star at solar metallicity (Hirschi et al. 2004; Ekström et al. 2012) can end its life in the BSG stage.

Furthermore, the rarity of BSG SNe (Pastorello et al. 2012, estimate that they represent $1-3 \%$ of all $\mathrm{CC} \mathrm{SNe)} \mathrm{suggests} \mathrm{that}$ the slightly low metal abundance is probably one of the ingredients or channels but not the only one. The main-sequence progenitor mass range that potentially gives rise to an exploding BSG is approximately 16-22 $M_{\odot}$ (Arnett et al. 1989 report this range for the progenitor of SN 1987A). Assuming an initial mass function (IMF) characterized by $\alpha=-2.35$ (Salpeter 1955), this corresponds to $\sim 15 \%$ of all CC SNe (here we adopted $8.5 M_{\odot}$ as the lower limit to produce a CC SN, see Smartt 2009). In order to match the rate presented by Pastorello et al. (2012), this $\sim 15 \%$ must be reduced by a factor of $\sim 10$, which is likely too high to be explained only in terms of moderately sub-solar metallicity.

Finally, we discuss the relatively weak association of BSG SNe to $\mathrm{H} \alpha$ emitting regions. BSG SNe were found to have a degree of associations to $\mathrm{HII}$ regions that is similar to those of SNe IIP and IIn, with many events sitting relatively far from bright star-forming regions. The association of a $\mathrm{SN}$ type to star-forming regions has been interpreted in terms of progenitor mass. The stronger the association, the larger is the typical initial progenitor mass (Anderson et al. 2012) of that SN type. However, since we know from modeling (e.g. Utrobin \& Chugai 2011; Taddia et al. 2012) that most of our BSG SNe arise from stars that are more massive $\left(M_{\mathrm{ZAMS}} \sim 20 M_{\odot}\right)$ than the typical RSG progenitors of SNe IIP $\left(\sim 10 M_{\odot}\right)$, it seems that this mass difference is too small to be detected by the NCR method (see also discussion in Crowther 2013). The weak association with H II regions for the massive progenitors of 1987A-like SNe could also be interpreted in terms of binary scenarios giving rise to longer lifetimes.

\section{Conclusions}

The main conclusions of this work are:

- SN 1987A-like events are either located in the outskirts of bright, metal rich galaxies or in faint, intrinsically metal poor galaxies.

- Most of the 1987A-like SNe show moderately sub-solar oxygen abundances $(\langle 12+\log (\mathrm{O} / \mathrm{H})\rangle=8.36 \pm 0.05 \mathrm{dex})$, comparable to that of the LMC. Compared to normal SNe IIP that arise from RSGs, they have a lower metallicity. The metallicity distribution of our BSG SNe is rather similar to that of $\mathrm{SNe}$ IIb.

- Two of our objects do indicate a solar or almost solar metallicity (SN 1998A and SN 2004em).
- BSG SNe show a moderate association to star-forming regions, similar to that of SNe IIP and IIn.

- The moderately low metallicity that characterizes the majority of the $\mathrm{SNe}$ in the sample likely plays a role in the formation of at least some 1987A-like SNe. This might be explained in the context of a single-star progenitor scenario. However, the nearly-solar abundances of two BSG SNe indicate that also other ingredients and/or channels (possibly binarity) contribute to produce some of these transients.

Acknowledgements. M.D. Stritzinger gratefully acknowledges generous support provided by the Danish Agency for Science and Technology and Innovation realized through a Sapere Aude Level 2 grant. G. Leloudas is supported by the Swedish Research Council through grant No. 623-2011-7117. The Dark Cosmology Centre is funded by the Danish National Research Foundation. The Oskar Klein Centre is funded by the Swedish Research Council. The Nordic Optical Telescop is operated by the Nordic Optical Telescope Scientific Association at the Observatorio del Roque de los Muchachos, La Palma, Spain, of the Instituto de Astrofisica de Canarias. This research has made use of the NASA/IPAC Extragalactic Database (NED) which is operated by the Jet Propulsion Laboratory, California Institute of Technology, under contract with the National Aeronautics and Space Administration. This research has made use of the SIMBAD database, operated at CDS, Strasbourg, France. We acknowledge the usage of the HyperLeda database.

\section{References}

Anderson, J. P., \& James, P. A. 2009, MNRAS, 399, 559

Anderson, J. P., Covarrubias, R. A., James, P. A., Hamuy, M., \& Habergham, S. M. 2010, MNRAS, 407, 2660

Anderson, J. P., Habergham, S. M., James, P. A., \& Hamuy, M. 2012, MNRAS, 424,1372

Arcavi, I., Gal-Yam, A., Cenko, S. B., et al. 2012, ApJ, 756, L30

Arnett, W. D. 1987, ApJ, 319, 136

Arnett, W. D., Bahcall, J. N, Kirshner, R. P, \& Woosley, S. E. 1989, ARA\&A, 27, 629

Asplund, M., Grevesse, N., Sauval, A. J., \& Scott, P. 2009, ARA\&A, 47, 481

Avila, G., Rupprecht, G., \& Beckers, J. M. 1997, Proc. SPIE, 2871, 1135

Barbon, R., Buondí, V., Cappellaro, E., \& Turatto, M. 1999, A\&AS, 139, 531

Boissier, S., \& Prantzos, N. 2009, A\&A, 503, 137

Bolton, A. S., Schlegel, D. J., Aubourg, É., et al. 2012, AJ, 144, 144

Bresolin, F., Gieren, W., Kudritzki, R.-P., et al. 2009, ApJ, 700, 309

Brunish, W. M., \& Truran, J. W. 1982, ApJS, 49, 447

Chen, T.-W., Smartt, S. J., Bresolin, F., et al. 2013, ApJ, 763, L28

Crowther, P. A. 2013, MNRAS, 428, 1927

de Vaucouleurs, G., de Vaucouleurs, A., Corwin, H. G., Jr., et al. 1991, Third Reference Catalogue of Bright Galaxies (New York: Springer)

Ekström, S., Georgy, C., Eggenberger, P., et al. 2012, A\&A, 537, A146

Eldridge, J. J. 2005, Ph.D. Thesis

Fransson, C., Cassatella, A., Gilmozzi, R., et al. 1989, ApJ, 336, 429

Garnett, D. R., Shields, G. A., Skillman, E. D., Sagan, S. P., \& Dufour, R. J. 1997, ApJ, 489, 63

Germany, L. M., Reiss, D. J., Schmidt, B. P., Stubbs, C. W., \& Suntzeff, N. B. 2004, A\&A, 415, 863

Gilmozzi, R., Cassatella, A., Clavel, J., et al. 1987, Nature, 328, 318

Hakobyan, A. A. 2008, Astrophys., 51, 69

Hakobyan, A. A., Mamon, G. A., Petrosian, A. R., Kunth, D., \& Turatto, M. 2009, A\&A, 508, 1259

Hakobyan, A. A., Adibekyan, V. Z., Aramyan, L. S., et al. 2012, A\&A, 544, A81 Hillebrandt, W., Hoeflich, P., Weiss, A., \& Truran, J. W. 1987, Nature, 327, 597 Hirschi, R., Meynet, G., \& Maeder, A. 2004, A\&A, 425, 649

Israel, F. P., Goss, W. M., \& Allen, R. J. 1975, A\&A, 40, 421

James, P. A., \& Anderson, J. P. 2006, A\&A, 453, 57

Jarrett, T. H., Chester, T., Cutri, R., Schneider, S. E., \& Huchra, J. P. 2003, AJ, 125,525

Kelly, P. L., \& Kirshner, R. P. 2012, ApJ, 759, 107

Kennicutt, R. C., Jr., Bresolin, F., \& Garnett, D. R. 2003, ApJ, 591, 801

Kewley, L. J., \& Ellison, S. L. 2008, ApJ, 681, 1183

Kleiser, I. K. W., Poznanski, D., Kasen, D., et al. 2011, MNRAS, 415, 372

Lang, D., Hogg, D. W., Mierle, K., Blanton, M., \& Roweis, S. 2010, AJ, 139, 1782

Langer, N. 1991, A\&A, 243, 155

Leloudas, G., Gallazzi, A., Sollerman, J., et al. 2011, A\&A, 530, A95

Modjaz, M., Kewley, L., Bloom, J. S., et al. 2011, ApJ, 731, L4 
F. Taddia et al.: A metallicity study of 1987A-like supernova host galaxies

Monet, D. G., Levine, S. E., Canzian, B, et al. 2003, AJ, 125, 984

Morris, T., \& Podsiadlowski, P. 2009, MNRAS, 399, 515

Nagao, T., Maiolino, R., \& Marconi, A. 2006, A\&A, 459, 85

Osterbrock, D. E. 1989, Research supported by the University of California, John Simon Guggenheim Memorial Foundation, University of Minnesota, et al.

(Mill Valley, CA: University Science Books), 422

Pagel, B. E. J., Edmunds, M. G., Fosbury, R. A. E., \& Webster, B. L. 1978, MNRAS, 184, 569

Pastorello, A., Baron, E., Branch, D., et al. 2005, MNRAS, 360, 950

Pastorello, A., Smartt, S. J., Botticella, M. T., et al. 2010, ApJ, 724, L16

Pastorello, A., Pumo, M. L., Navasardyan, H., et al. 2012, A\&A, 537, A141

Perez, J., Michel-Dansac, L., \& Tissera, P. B. 2011, MNRAS, 417, 580

Pettini, M., \& Pagel, B. E. J. 2004, MNRAS, 348, L59

Pilyugin, L. S., \& Thuan, T. X. 2007, ApJ, 669, 299

Pilyugin, L. S., Vílchez, J. M., \& Contini, T. 2004, A\&A, 425, 849

Podsiadlowski, P. 1992, PASP, 104, 717

Russell, S. C., \& Dopita, M. A. 1990, ApJS, 74, 93

Saio, H., Nomoto, K., \& Kato, M. 1988, Nature, 334, 508
Salpeter, E. E. 1955, ApJ, 121, 161

Sanders, N. E., Soderberg, A. M., Levesque, E. M., et al. 2012, ApJ, 758, 132

Schlegel, D. J., Finkbeiner, D. P., \& Davis, M. 1998, ApJ, 500, 525

Smartt, S. J. 2009, ARA\&A, 47, 63

Spergel, D. N., Bean, R., Doré, O., et al. 2007, ApJS, 170, 377

Stanishev, V. 2007, Astron. Nachr., 328, 948

Stoll, R., Prieto, J. L., Stanek, K. Z., \& Pogge, R. W. 2013, ApJ, 773, 12

Taddia, F., Stritzinger, M. D., Sollerman, J., et al. 2012, A\&A, 537, A140

Taubenberger, S., Navasardyan, H., Maurer, J. I., et al. 2011, MNRAS, 413, 2140

Thöne, C. C., Michałowski, M. J., Leloudas, G., et al. 2009, ApJ, 698, 1307

Tremonti, C. A., Heckman, T. M., Kauffmann, G., et al. 2004, ApJ, 613, 898

Utrobin, V. P., \& Chugai, N. N. 2011, A\&A, 532, A100

van den Bergh, S. 1988, Proc. Astron. Soc. Aust., 7, 443

van den Bergh, S., \& McClure, R. D. 1989, ApJ, 347, L29

Weiss, A., Hillebrandt, W., \& Truran, J. W. 1988, A\&A, 197, L11

Woosley, S. E. 1988, ApJ, 330, 218

Woosley, S. E., Heger, A., \& Weaver, T. A. 2002, Rev. Mod. Phys., 74, 1015

Pages 14 to 24 are available in the electronic edition of the journal at http: //www . aanda. org 

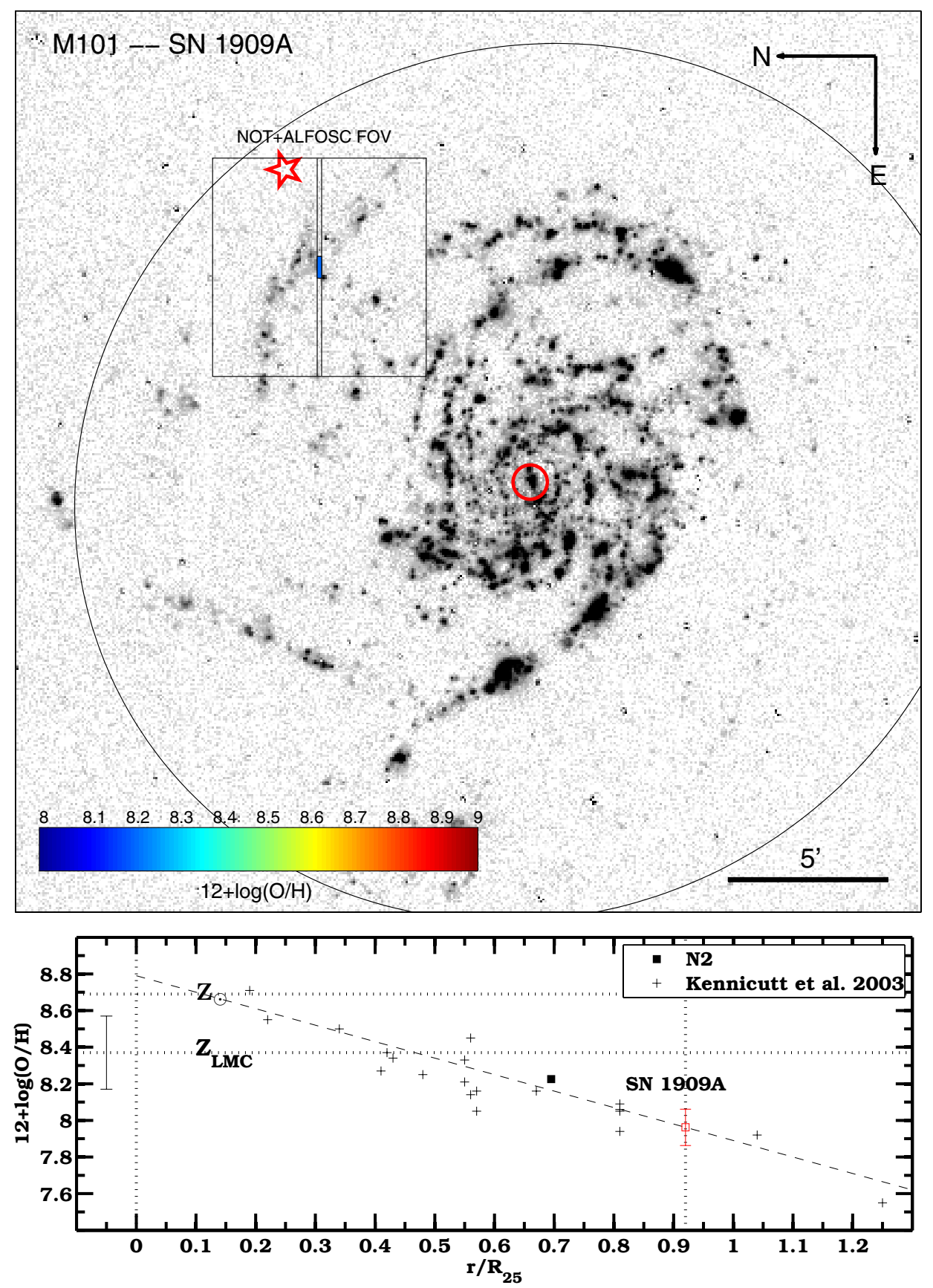

Fig. 4. (Top panel) Continuum-subtracted $\mathrm{H} \alpha$ image of M 101, obtained from http://www . aoc.nrao. edu. The 25th $B$-band magnitude elliptic contour is shown by a black solid line, along with the position of SN 1909A (marked by a red star) and the center of the galaxy (marked by a red circle). We also show the field of view (FOV) of ALFOSC and the slit position. The width of the slit ( $\left.1^{\prime \prime}\right)$ has been enhanced in the figure to show, through a color code, the measured metallicity at the position of a bright H II region that we inspected. (Bottom panel) Metallicity gradient of M 101 from Kennicutt et al. (2003). In the plot we include our measurement, which matches with those that are already published. The linear fit from Kennicutt et al. (2003) is shown by a dashed line. The interpolated metallicity at the SN distance is marked by a red square and its uncertainty corresponds to the fit error. The error bar $( \pm 0.2 \mathrm{dex})$ for our $\mathrm{N} 2$ measurement is shown aside. The positions of $\mathrm{SN}$ and nucleus are marked by vertical dotted lines. The solar metallicity (Asplund et al. 2009) and the LMC metallicity (Russell \& Dopita 1990) are indicated by two horizontal dotted lines. 
F. Taddia et al.: A metallicity study of 1987A-like supernova host galaxies
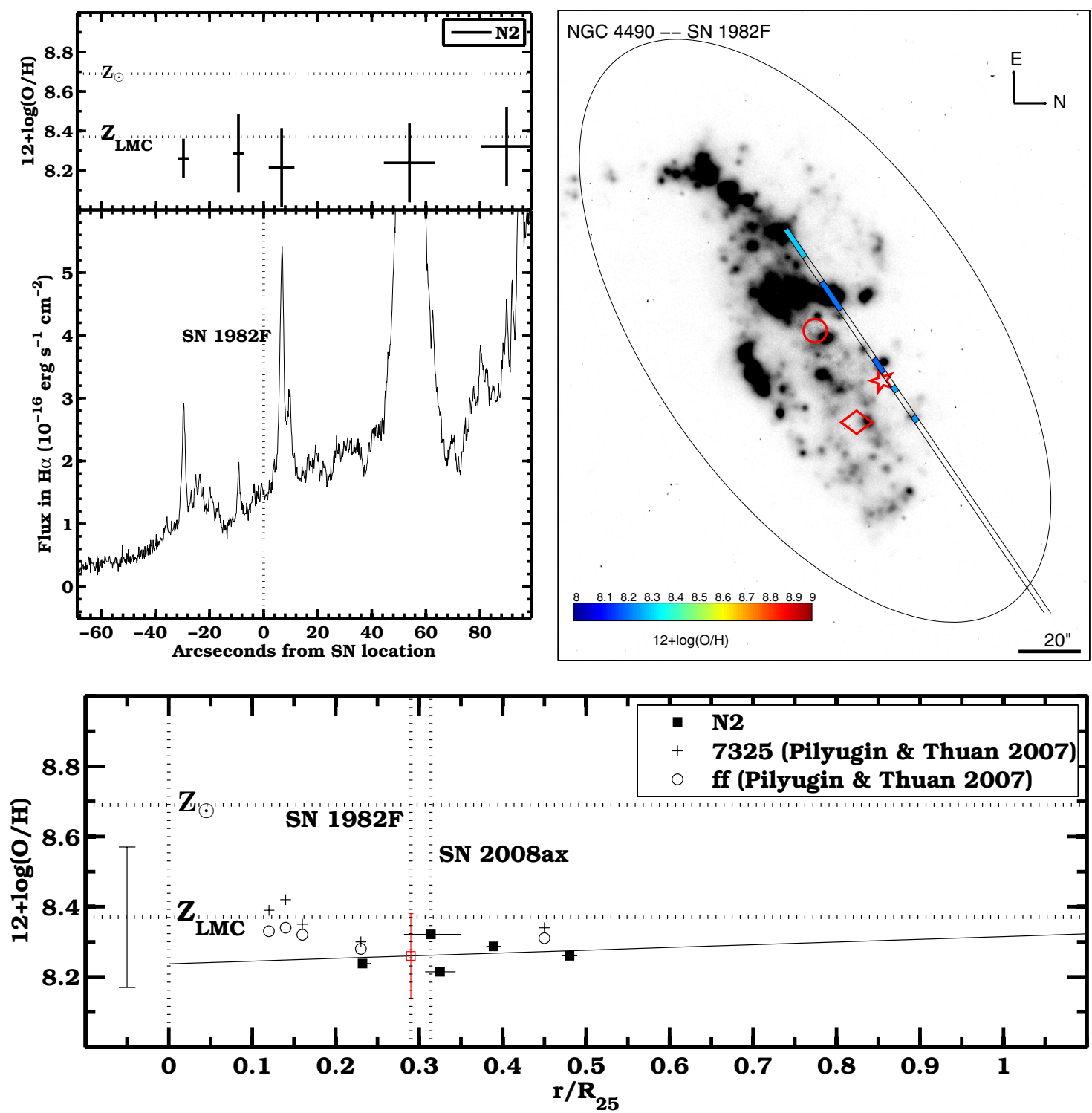

Fig. 5. (Top-right panel) Continuum-subtracted H $\alpha$ image of NGC 4490. The 25th $B$-band magnitude elliptic contour is shown by a black solid line, along with the positions of SN 1982F (marked by a red star), SN 2008ax (marked by a red diamond) and the center of the galaxy (marked by a red circle). The slit position is shown, and a color code is used to present the N2 metallicity measurements at the position of each $\mathrm{H}$ II region that we inspected. The slit width ( $\left.1^{\prime \prime}\right)$ has been enhanced to better show the colored patches. (Top-left panel) Flux at the H $\alpha$ wavelength along the slit, shown as a function of the distance from the location of SN 1982F (marked by a dashed line). The N2 measurements are shown at the corresponding positions in the upper sub-panel. (Bottom panel) Metallicity gradient of NGC 4490. In the plot we include the measurements from Pilyugin \& Thuan (2007), which match our N2 metallicity estimates. The linear fit on our measurements is shown by a solid line. The interpolated metallicity at the distance of SN $1982 \mathrm{~F}$ is marked by a red square and its uncertainty corresponds to the fit error. The error bar ( \pm 0.2 dex) for our N2 measurements is shown aside. The positions of SN 1982F, SN 2008ax and nucleus are marked by vertical dotted lines. The solar metallicity (Asplund et al. 2009) and the LMC metallicity (Russell \& Dopita 1990) are indicated by two horizontal dotted lines. 

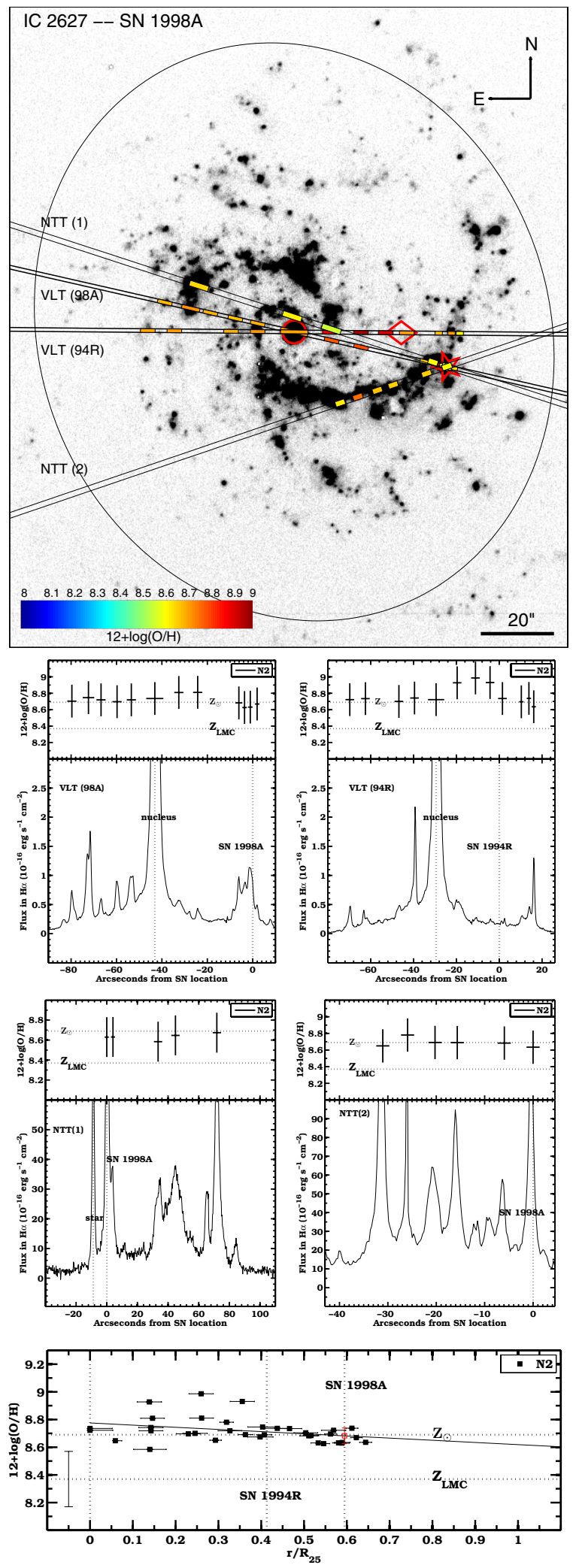

Fig. 6. (Top panel) Continuum-subtracted $\mathrm{H} \alpha$ image of IC 2627. The 25th $B$-band magnitude elliptic contour is shown by a black solid line, along with the position of SN 1998A (marked by a red star), the center of the galaxy (marked by a red circle) and the position of SN 1994R (marked by a red diamond). The positions of the four slits are shown, and a color code is used to present the N2 metallicity measurements at the position of each H II region that we inspected. (Center panels) Flux at the $\mathrm{H} \alpha$ wavelength along the four slits, shown as a function of the distance from the SN location. These positions are marked by dotted lines. The N2 measurements for each H II region are shown at the corresponding positions in the upper sub-panels. (Bottom panel) Metallicity gradient of IC 2627. The linear fit on our measurements is shown by a solid line. The interpolated metallicity at the $\mathrm{SN}$ distance is marked by a red square and its uncertainty corresponds to the fit error. The error bar ( \pm 0.2 dex $)$ for our N2 measurements is shown aside. The positions of SN and nucleus are marked by vertical dotted lines. The solar metallicity (Asplund et al. 2009) and the LMC metallicity (Russell \& Dopita 1990) are indicated by two horizontal dotted lines. 
F. Taddia et al.: A metallicity study of 1987A-like supernova host galaxies
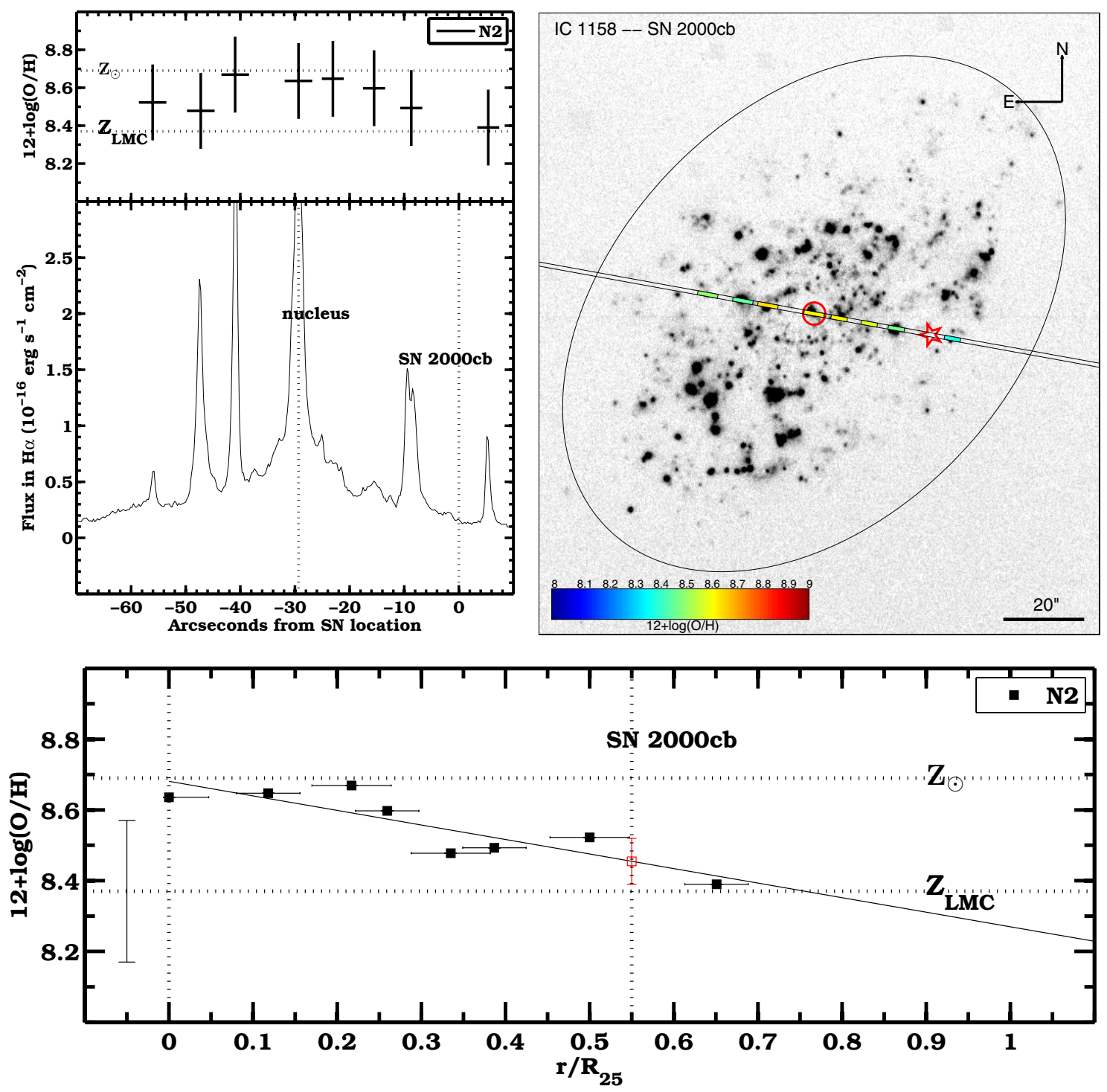

Fig. 7. (Top-right panel) Continuum-subtracted $\mathrm{H} \alpha$ image of IC 1158 . The 25th $B$-band magnitude elliptic contour is shown by a black solid line, along with the position of SN 2000cb (marked by a red star) and the center of the galaxy (marked by a red circle). The slit position is shown, and a color code is used to present the N2 metallicity measurements at the position of each H II region that we inspected. (Top-left panel) Flux at the $\mathrm{H} \alpha$ wavelength along the slit, shown as a function of the distance from the SN location (marked by a dotted line, like the nucleus position). The N2 measurements are shown at the corresponding positions in the top sub-panel. (Bottom panel) Metallicity gradient IC 1158, see the bottom-panel caption of Fig. 6 for details. 

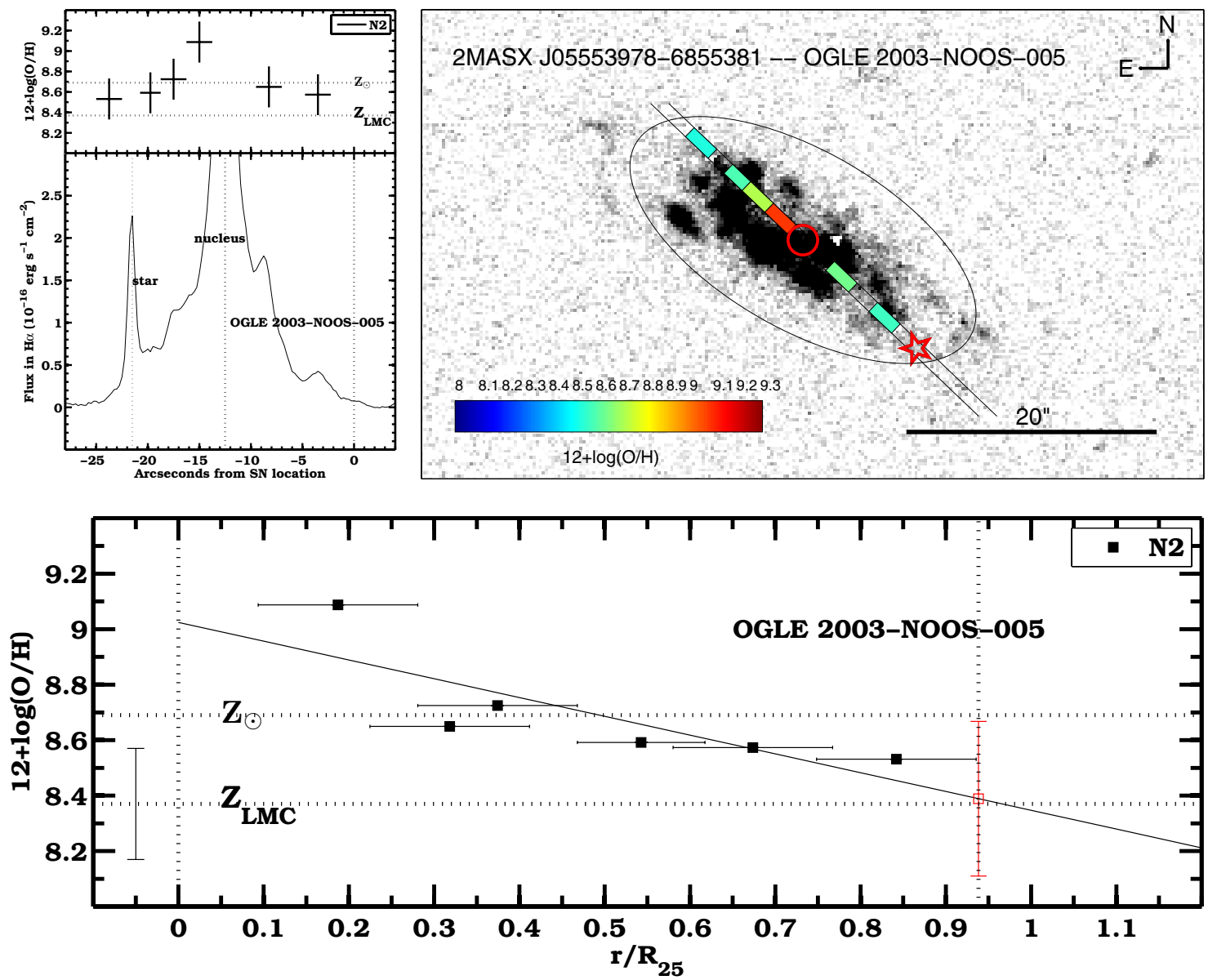

Fig. 8. (Top-right panel) Continuum-subtracted H $\alpha$ image of 2MASX J05553978-6855381, see the top-right panel caption of Fig. 7 for details. (Top-left panel) Flux at the $\mathrm{H} \alpha$ wavelength along the slit, see the top-left panel caption of Fig. 7 for details. (Bottom panel) Metallicity gradient of 2MASX J05553978-6855381, see the bottom-panel caption of Fig. 6 for details. 
F. Taddia et al.: A metallicity study of 1987A-like supernova host galaxies
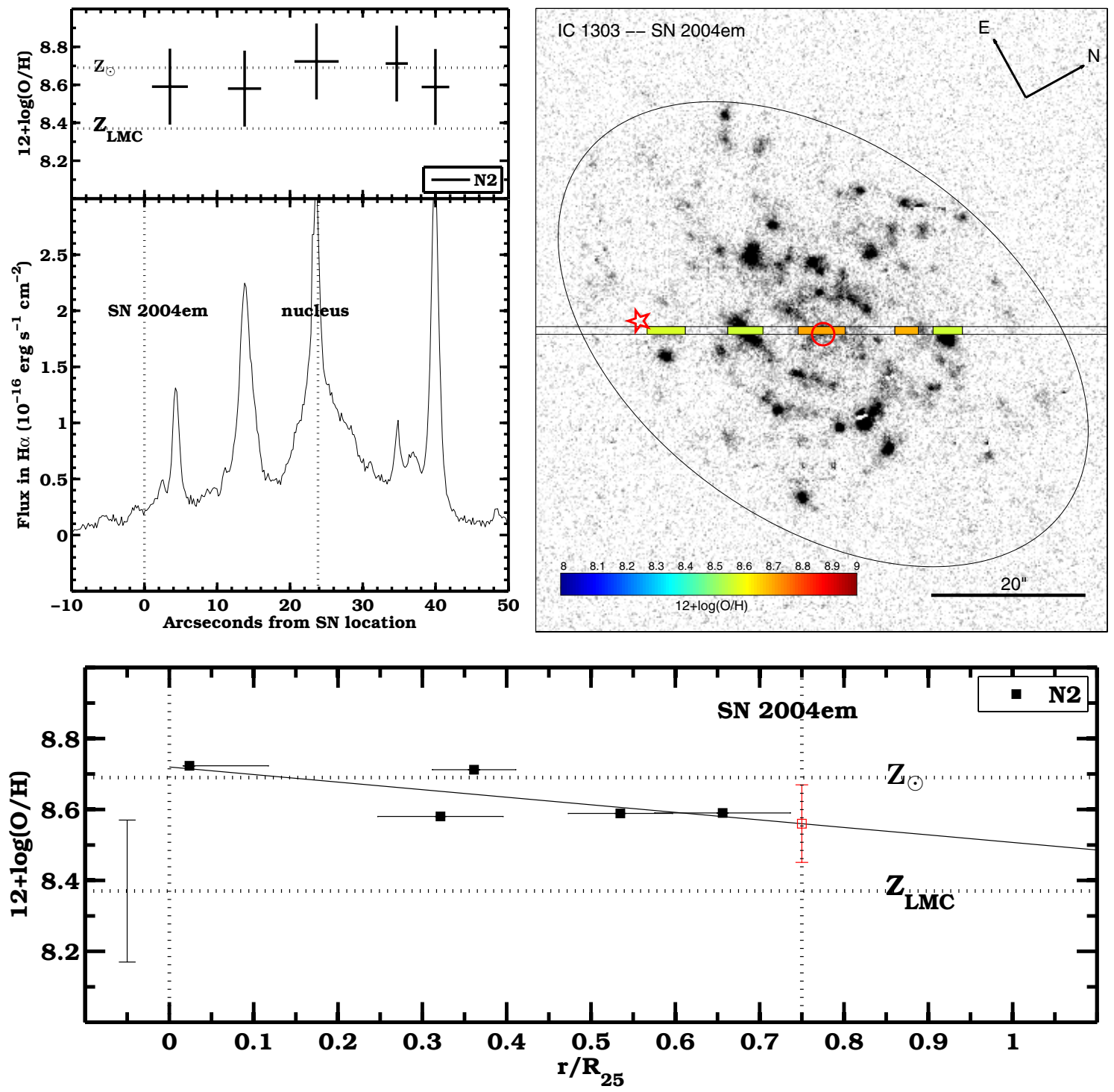

Fig. 9. (Top-right panel) Continuum-subtracted $\mathrm{H} \alpha$ image of IC 1303, see the top-right panel caption of Fig. 7 for details. (Top-left panel) Flux at the $\mathrm{H} \alpha$ wavelength along the slit, see the top-left panel caption of Fig. 7 for details. (Bottom panel) Metallicity gradient of IC 1303, see the bottom-panel caption of Fig. 6 for details. 

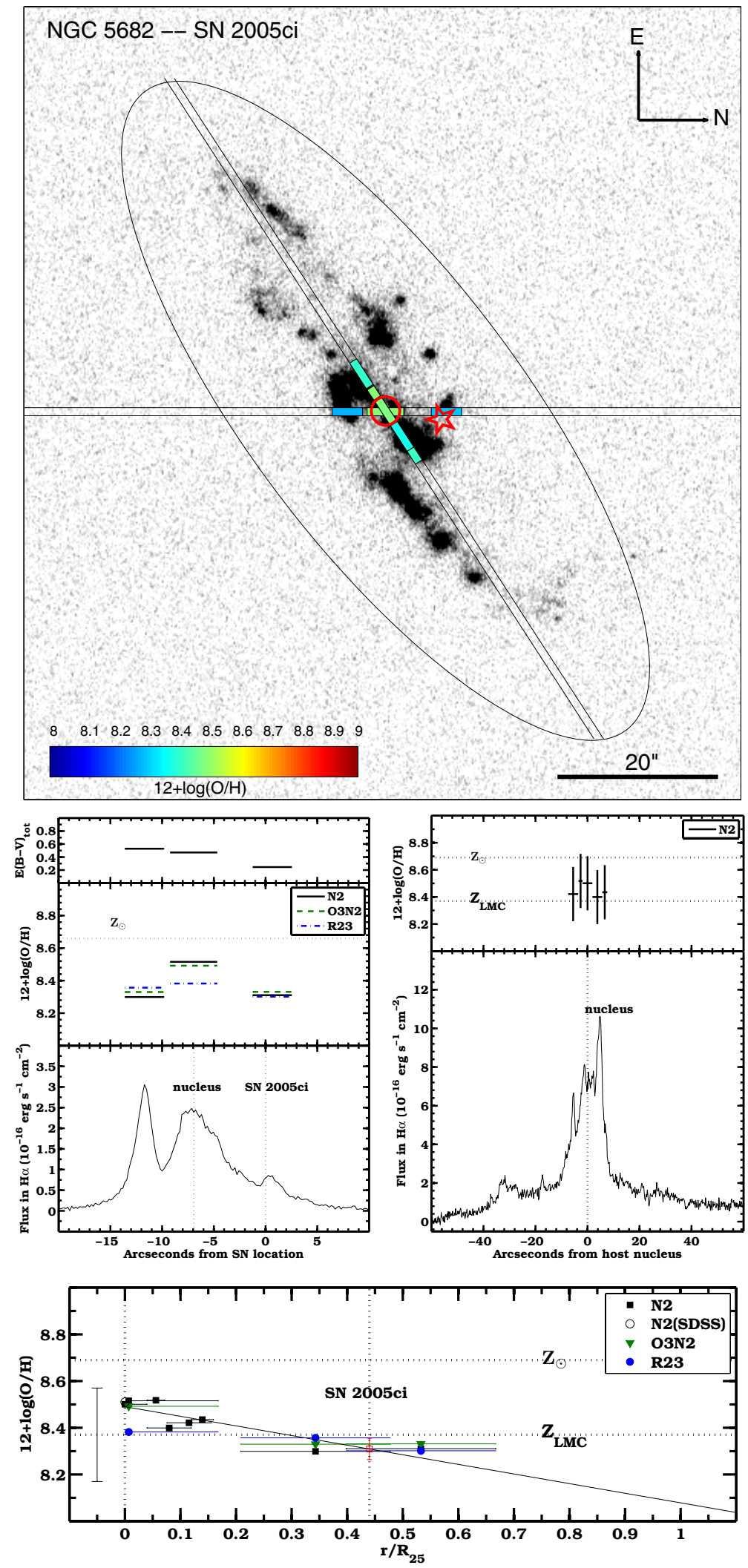

Fig. 10. (Top panel) Continuum-subtracted H $\alpha$ image of NGC 5682, see the top-right panel caption of Fig. 7 for details. Here we show the positions of two slits. (Center panels) Flux at the $\mathrm{H} \alpha$ wavelength along the two slits, shown as a function of the distance from the SN location and from the nucleus. These positions are marked by vertical dotted lines. The N2 measurements for each $\mathrm{H}$ II region are shown at the corresponding positions in the top sub-panels. For the slit at the SN position we also show the O3N2 and $R 23$ metallicity estimates, and the $E(B-V)$ values that we computed through the measured Balmer decrements. (Bottom panel) Metallicity gradient of NGC 5682, see the bottom-panel caption of Fig. 6 for details. We also reported the N2 metallicity from the spectrum taken by SDSS at the center of the galaxy. 
F. Taddia et al.: A metallicity study of 1987A-like supernova host galaxies
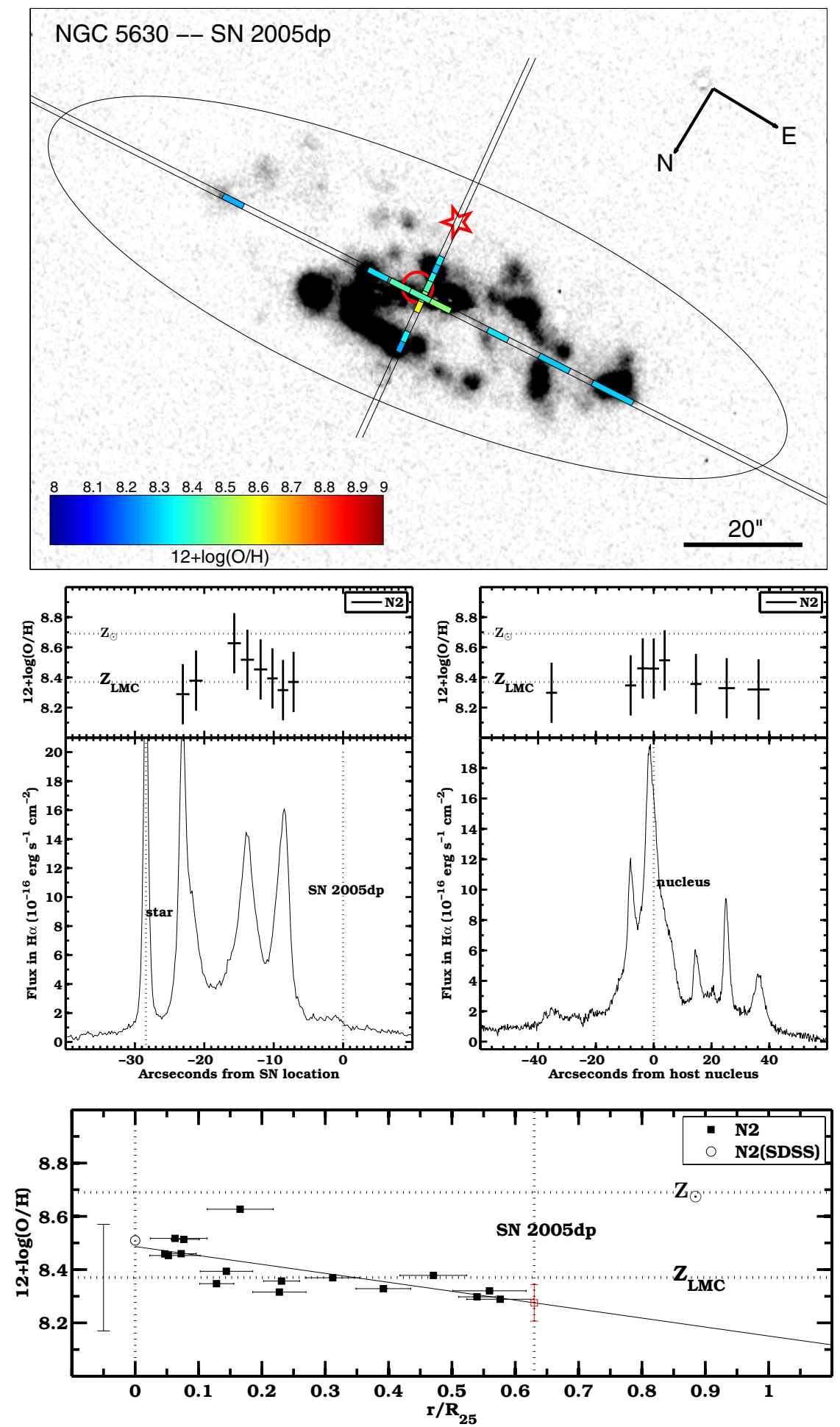

Fig. 11. (Top panel) Continuum-subtracted H $\alpha$ image of NGC 5630, see the top-right panel caption of Fig. 7 for details. Here we show the positions of two slits. (Center panels) Flux at the $\mathrm{H} \alpha$ wavelength along the two slits, shown as a function of the distance from the SN location and from the nucleus. See the top-left panel caption of Fig. 7 for details. (Bottom panel) Metallicity gradient of NGC 5630, see the bottom-panel caption of Fig. 10 for details. 

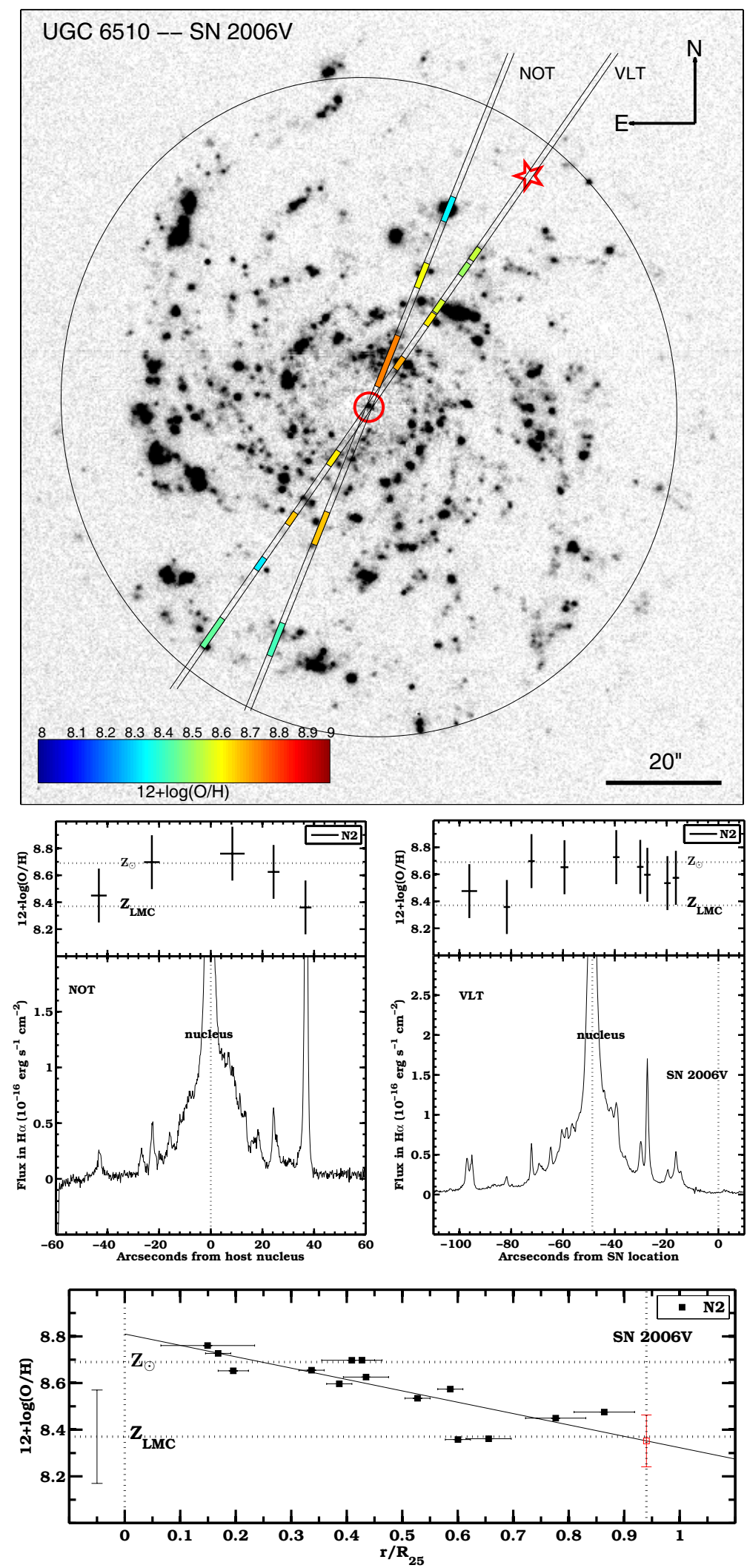

Fig. 12. (Top panel) Continuum-subtracted $\mathrm{H} \alpha$ image of UGC 6510, see the top-right panel caption of Fig. 7 for details. Here we show the positions of two slits, one for the NOT and one for the VLT spectrum. (Center panels) Flux at the H $\alpha$ wavelength along the two slits, shown as a function of the distance from the SN location and from the nucleus. See the top-left panel caption of Fig. 7 for details. (Bottom panel) Metallicity gradient of UGC 6510, see the bottom-panel caption of Fig. 6 for details. 
F. Taddia et al.: A metallicity study of 1987A-like supernova host galaxies
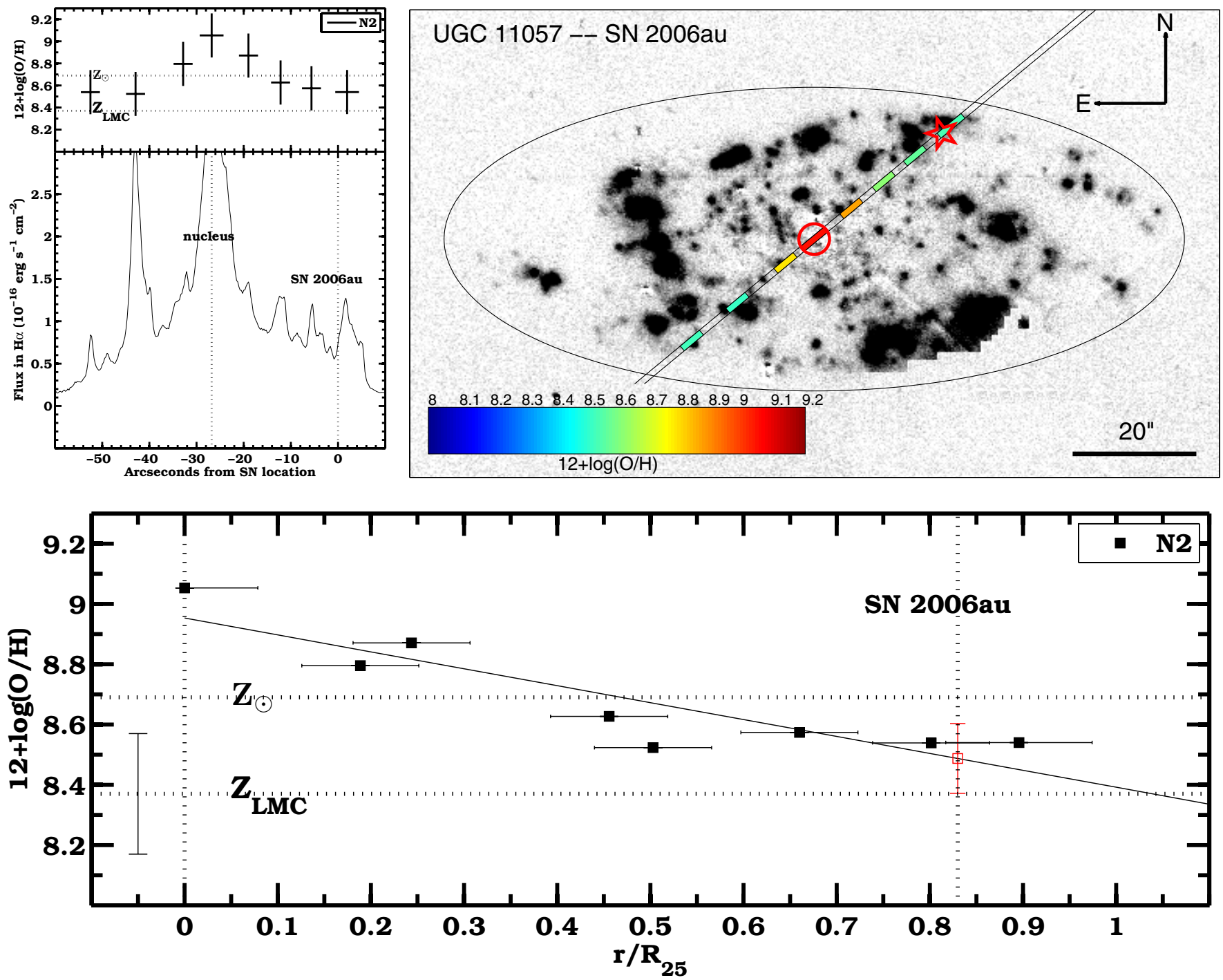

Fig. 13. (Top-right panel) Continuum-subtracted H $\alpha$ image of UGC 11057, see the top-right panel caption of Fig. 7 for details. (Top-left panel) Flux at the $\mathrm{H} \alpha$ wavelength along the slit, see the top-left panel caption of Fig. 7 for details. (Bottom panel) Metallicity gradient of UGC 11057, see the bottom-panel caption of Fig. 7 for details. 

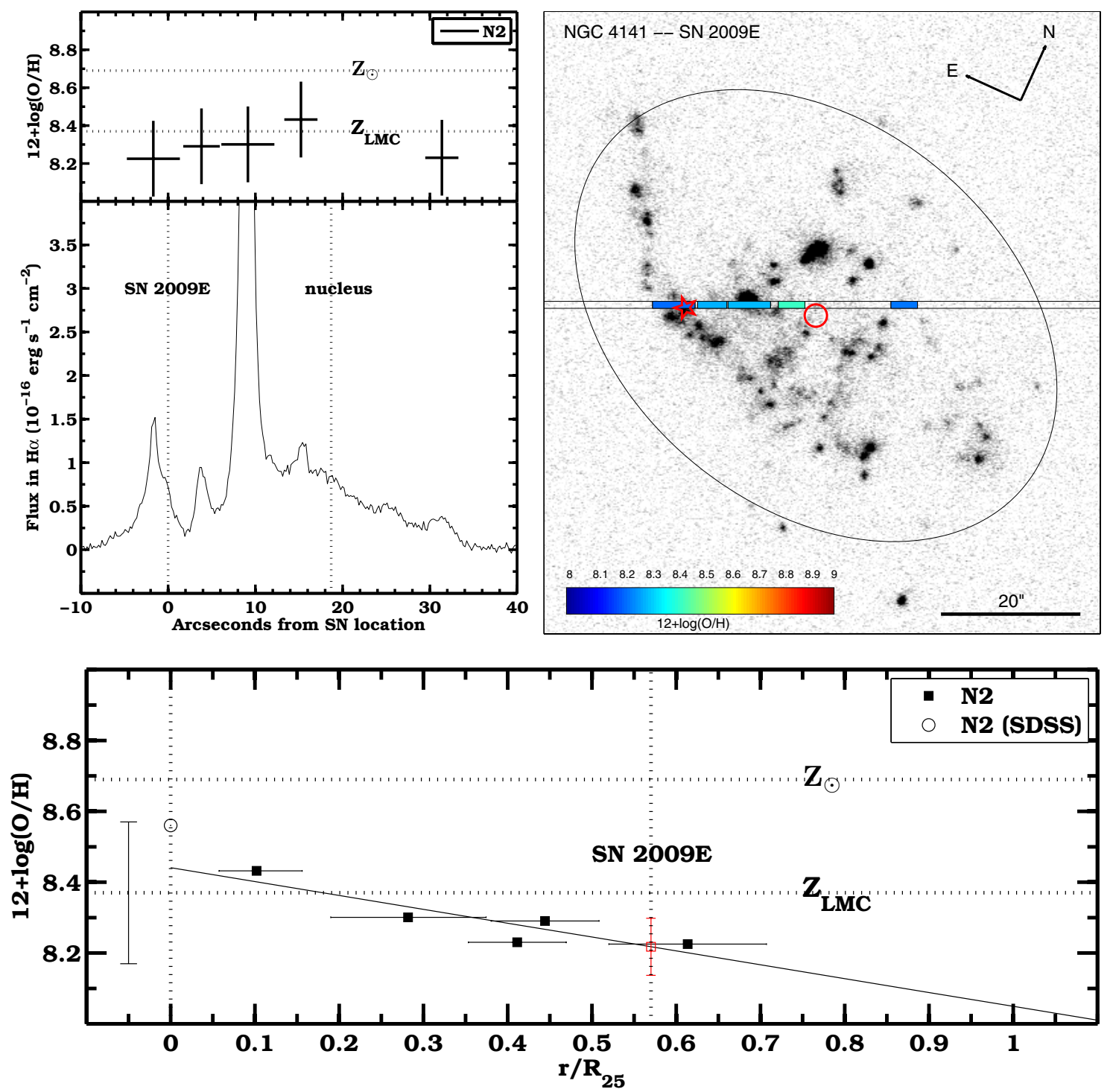

Fig. 14. (Top-right panel) Continuum-subtracted $\mathrm{H} \alpha$ image of NGC 4141, see the top-right panel caption of Fig. 7 for details. (Top-left panel) Flux at the $\mathrm{H} \alpha$ wavelength along the slit, see the top-left panel caption of Fig. 7 for details. (Bottom panel) Metallicity gradient of NGC 4141, see the bottom-panel caption of Fig. 10 for details. 